\title{
Ultra-High-Throughput Acoustic Droplet Ejection-Open Port Interface-Mass Spectrometry for Parallel Medicinal Chemistry
}

Kenneth J. DiRico, ${ }^{1, \dagger}$ Wenyi Hua, ${ }^{1, \dagger}$ Chang Liu, ${ }^{2}$ Joseph W. Tucker, ${ }^{1}$ Anokha S. Ratnayake, ${ }^{1}$ Mark E. Flanagan, ${ }^{1}$ Matthew D. Troutman, ${ }^{1}$ Mark C. Noe,${ }^{1}$ and Hui Zhang ${ }^{1, *}$

\author{
Affiliations: \\ ${ }^{1}$ Pfizer Global Research and Development, Eastern Point Road, Groton, CT, 06340 USA. \\ ${ }^{2}$ SCIEX, 71 Four Valley Drive, Concord, ON, L4K 4V8 Canada. \\ $\dagger$ These authors contributed equally to this work. \\ * Correspondence to:
}

Hui Zhang, Hui.Zhang3@pfizer.com

\section{Supplemental materials, methods and figures}

All reagents were used as purchased from commercial suppliers. Solvents, purchased from SigmaAldrich, were anhydrous, Sure/Seal ${ }^{\mathrm{TM}}$ grade, and were used with no further purification. The palladium catalysts and bases were purchased from commercial sources and stored in a glovebox. All reactions, including parallel syntheses, were performed inside an MBraun glovebox (oxygen level $<10 \mathrm{ppm})$.

$\underline{\text { Abbreviations }}$

Dimethyl sulfoxide (DMSO)

Dimethyl acetamide (DMAc)

$N$-Ethyl- $N$ '-[3-(dimethylamino)propyl]carbodiimide hydrochloride (EDC-HCl)

2-Hydroxypyridine $\mathrm{N}$-oxide (HOPO)

1-[Bis(dimethylamino)methylene]-1H-1,2,3-triazolo[4,5-b]pyridinium 3-oxide hexafluorophosphate (HATU)

Base

$N, N$-Diisopropylethylamine (Hunig's base, DIEA)

1,8-Diazabicyclo[5.4. 0]undec-7-ene (DBU)

1,3,4,6,7,8-Hexahydro-1-methyl-2H-pyrimido[1,2-a]pyrimidine (MTBD) 
2-tert-Butyl-1,1,3,3-tetramethylguanidine (BTMG)

2-tert-Butylimino-2-diethylamino-1,3-dimethylperhydro-1,3,2-diazaphosphorine (BEMP) tert-Butylimino-tri(pyrrolidino)phosphorane (BTTP)

1-Ethyl-2,2,4,4,4-pentakis(dimethylamino)- $2 \lambda^{5}, 4 \lambda^{5}$-catenadi(phosphazene) (P2-Et)

Catalyst

Methanesulfonato 1,1-ferrocenediyl-bis(diphenylphosphino) (2'-amino-1,1'-biphenyl-2-yl) palladium(II) (dppf Pd G3)

[(4,5-Bis(diphenylphosphino)-9,9-dimethylxanthene)-2-(2'-amino-1,1'-biphenyl)]palladium(II) methanesulfonate (XantPhos Pd G3)

(2-Dicyclohexylphosphino-2',6'-diisopropoxy-1,1'-biphenyl)[2-(2'-amino-1,1'-

biphenyl)]palladium(II) methanesulfonate (Ruphos Pd G3)

[(2-Di-tert-butylphosphino-2',4',6'-triisopropyl-1,1'-biphenyl)-2-(2'-amino-1,1'-biphenyl)] palladium(II) methanesulfonate ( $t$ BuXPhos-Pd-G3)

[2-(Di-1-adamantylphosphino)-2',4',6'-triisopropyl-3,6-dimethoxybiphenyl][2-(2'-amino-1,1'biphenyl)]palladium(II) methanesulfonate (AdBrettPhos Pd G3)

[(2-Di-tert-butylphosphino-3,6-dimethoxy-2',4',6'-triisopropyl-1,1'-biphenyl)-2-(2'-amino-1,1'biphenyl)]palladium(II) methanesulfonate (tBuBrettPhos Pd G3)

tert-BuBrettPhos-Pd-G3, [(2-Di-tert-butylphosphino-3,6-dimethoxy-2',4',6'-triisopropyl-1,1'biphenyl)-2-(2'-amino-1,1'-biphenyl)]palladium(II) methanesulfonate (tBuBrettPhos Pd G3)

[(2-Di-cyclohexylphosphino-3,6-dimethoxy-2',4',6'- triisopropyl-1,1'-biphenyl)-2-(2'-amino-1,1' -biphenyl)]palladium(II) methanesulfonate methanesulfonate (BrettPhos Pd G3)

( \pm ) [1,1'-Binaphthalene]-2,2'-diylbis[diphenylphosphine]palladium(II) methanesulfonate methanesulfonate (rac-BINAP-Pd-G3)

[(tri-tert-butylphosphine)]palladium(II) methanesulfonate methanesulfonate $\left(\mathrm{P}(t-\mathrm{Bu})_{3} \mathrm{Pd} \mathrm{G} 3\right)$

Methanesulfonato(1,3,5,7-tetramethyl-8-phenyl-2,4,6-trioxa-8-phosphaadamantane)(2'-amino1,1'-biphenyl-2-yl)palladium(II) (PPA G3)

[4-(Di-tert-butylphosphino)- $N, N$-dimethylaniline-2-(2'-aminobiphenyl)]palladium(II)

methanesulfonate (Aphos Pd G3)

(2-Dicyclohexylphosphino-2', 4', 6'-triisopropyl-1,1'-biphenyl)[2-(2'-amino-1,1'biphenyl)]palladium(II) methanesulfonate (Xphos Pd G3)

[1,1'-Bis(di-tert-butylphosphino)ferrocene] palladium(II) methanesulfonate methanesulfonate (DTBTF Pd G3) 
[(R)-1-[(Sp)-2-(Dicyclohexylphosphino)ferrocenyl] ethyldi-tert-butylphosphine][2-(2'-amino1,1'-biphenyl)]palladium(II) methanesulfonate (Josiphos Pd G3)

(2-(Di-1-adamantylphosphino)morpholinobenzene)[2-(2'-amino-1,1'-biphenyl)]palladium(II) methanesulfonate (Mordalphos Pd G3)

[(2-Di-tert-butylphosphino-3-methoxy-6-methyl-2',4',6'-triisopropyl-1,1'-biphenyl)-2-(2aminobiphenyl)]palladium(II) methanesulfonate (Rockphos Pd G3)

Nanomolar-scale chemistry experiments

Nanoscale reactions (200 nmol) were run using Labcyte 384-well plates (Cat. No. LP0200, Cyclic Olefin-Copolymer COC, $12.5 \mu \mathrm{L}$-well plates, flat-bottom, clear) as reaction plates. Advantage 384-well plates (Analytical Sales and Services, Cat. No. 38120, polypropylene, $120 \mu \mathrm{L}$-wells, flatbottom, clear) were used as stock solution source plates and Labcyte 384-well plates (Cat. No. LV188 COC, $40 \mu \mathrm{L}$-wells, round ${ }^{\mathrm{TM}}$-bottom, black) were used as analytical plates on both UPLCMS and ADP-OPI-MS equipment. Dosing of the 384-well plates was accomplished in the glovebox using a Mosquito HTS liquid handling robot (TTP Labtech, $4.5 \mathrm{~mm}$ pitch tip spool) and TTP Labtech software. After dosing, the 384-well plates were covered with a perfluoro alkoxy alkane (PFA) mat (Analytical Sales and Services, contact for Cat. No.), followed by a silicon rubber mat (Analytical Sales and Services, contact for Cat. No. ) and secured in a Nano Nest (Analytical Sales and Services, contact for Cat. No.).

When heating was required, the Nano Nest was placed in an oven with $\mathrm{N}_{2}$ airflow (Fisher Scientific Isotemp model 280A).

\section{UPLC-MS Analytical Analysis}

Reactions were analyzed using an Agilent UPLC-MS (Model No. 1290 UPLC, coupled with a $6150 \mathrm{MS}$ ). The LC system was equipped with a G7120A high-speed binary pump, G7117A diode array detector, G7167B multisampler, and G7116B thermostated column compartment. Column: Aquity UPLC BEH C18, $1.7 \mu \mathrm{m}, 2.1$ x $30 \mathrm{~mm}$ (Part No. 186002349). Mobile phase A: water (Fisher Optima LC/MS grade) with $0.1 \%$ formic acid; Mobile phase B: acetonitrile (Fisher Optima LC/MS grade) with $0.1 \%$ formic acid. The MS detector was equipped with an electrospray ionization (ESI) source in positive and negative mode. High-throughput batch data analysis to produce Excel spreadsheets was done with Virscidian analytical Studio (ASPro) software (v. 10.7).

The ADE-OPI-MS system:

The ADE-OPI-MS platform shown in Figure 1 consists of an standard ATS Gen 4+ acoustic dispensing system (EDC Biosystems, Fremont, CA), an open-port probe sampling interface connected to both a carrier solvent pump and a transfer capillary leading to the standard IonDriveTM Turbo V ESI source of a Sciex Triple QuadTM 6500+ System (for experiment one) or Sciex TripleTOF® 6600 (for experiment two and three). The rotation function of the targetplate gripper on the ATS system was disabled. A 3-D printed OPI-holder with external dimensions of a standard microplate was loaded in the target-plate gripper to position the open 
port capture region with a gap of $2 \mathrm{~mm}$ above the source microplate. The $\mathrm{x}-\mathrm{y}$ position of the OPI was set in software to align the OPI capture region to the acoustic transducer axis. In a separate step, the source well is also aligned to the acoustic transducer axis. The ATS-100 software was used to control acoustic ejection. The mass spectrometer was operated with standard software (SCIEX Analyst 1.6.2). Peakview V1.2 with BioTools V1.0 companion feature was used to view and process MS data. Visualization of plate heat map was generated using Virscidian ASPro software (v. 10.7).

\section{Experimental}

Experiment 1. Palladium-catalyzed C-N coupling reactions of 4-phenylpiperidine and 3bromopyridine at nanomolar scale $(2 \mu \mathrm{L}$ volume $)$ in a 384-well plate in a 6 x 16 format.

Stock solutions of each of the reaction components were made as follows: 4-phenylpiperidine (0.6 $\mathrm{M}$ in DMSO), 3-bromopyridine (0.4 M in DMSO), six organic superbases (0.8 M in DMSO) and sixteen Pd-precatalyst (0.04 M in DMSO). Each of the solutions was dispensed in $50 \mu \mathrm{L}$ charges to a 384-well source plate.

The Mosquito robotics instrument was used to combine the source plate solutions by multiaspiration of $500 \mathrm{~nL}$ each of the 3-bromopyridine and catalyst solutions, which were then dispensed to an appropriate low-volume 384-well reaction plate. Multi-aspiration was repeated with $500 \mathrm{~nL}$ each of 4-phenylpiperidine and base; this was also dispensed to the appropriate reaction well. On addition of the catalyst/base, the well contents were mixed three times, with pipette tips moving $1 \mathrm{~mm}$ per dispense, in order to fully mix components. The final reaction concentrations were $0.1 \mathrm{M}$ in a $2 \mu \mathrm{L}$ volume. Once the 384-well plate was fully dosed, it was sealed in a Nano Nest, then allowed to sit at room temperature for 18 hours. Using an Eppendorf multi pipette, the reaction was then quenched with $2 \mu \mathrm{L}$ of a DMSO stock solution of $5 \%$ glacial acetic acid and biphenyl (resulting in $3 \mathrm{~mol} \%$ biphenyl relative to 4-phenylpiperidine), and further diluted with DMSO for a total volume of $10 \mu \mathrm{L}(20 \mathrm{mM})$. Transfer of $1 \mu \mathrm{L}$ to a 384-well analytical COC (Auroro, black, round-bottom, $40 \mu \mathrm{L}$ volume) microplate containing $19 \mu \mathrm{L}$ of DMSO provided a final volume of $20 \mu \mathrm{L}$ (1 $\mathrm{mM}$ solution). The plate was then analyzed by ADE-OPIMS followed by UPLC-MS on an Agilent 1290/6150. For ADE-OPI-MS detection, 5nL samples were injected and analyzed with pseudo MRM mode (with parent-to-parent transition).For the UPLC-MS data batch analytics, the ratio of the UV254 area counts of product over internal standard was used to compare the relative performance of the reactions. Alternatively, the ratio of the UPLC-MS UV254 area counts of product compared to the UV254 LC area counts of the remaining starting material could be used to directly assess the relative performance of the reactions. Plate mapping and the raw data containing the LC area ratios for the 384-well plate can be found in Supplemental Table 2. The graphical representation of this data is shown in Supplemental Figure 5. 
Experiment 2. Amide formation: reaction of two amine templates with 96 acids using EDC/HOPO as the coupling agent (see Supplemental Figure 6).

A 384-well plate experiment examining the reactivity of two secondary amine templates under dehydrating coupling conditions was run at 200 nanomolar scale; dosing was carried out from a 384-well source plate containing stock solutions of starting materials and reagents into a 384-well plate by a Mosquito $\mathrm{nL}$ pipetting liquid handler. ${ }^{1}$

The 96 acid monomers were first formatted as stock solutions $(0.45 \mathrm{M}$ in DMAc, $30 \mu \mathrm{L}$ volume each), then dispensed to a 384-well low-volume plate (Labcyte, Cat. No. LP0200) by a Mosquito liquid handler in $1 \mu \mathrm{L}$ doses laid out in two 96-well patterns: wells A1-D24 contained monomers for reaction with template 1, and wells E1-H24 held monomers for reaction with template 2 (Supplemental Table 3). To a 384-well source plate (Analytical Sales and Services, Cat. No. 38120) were added the two template amines [0.4 M in DMAc with DIEA (5 equiv.)], in columns 1 and 2, respectively. A combined stock solution of $\mathrm{EDC} / \mathrm{HOPO}(2: 1,0.52 \mathrm{M}$, relative to the amine in DMAc) was put into column 3 of the 384-well source plate for dosing to the reaction plate via a Mosquito HTS liquid handler. The $\mathrm{nL}$ liquid handler added the stock solutions of the templates and coupling reagents by multi-aspiration of $500 \mathrm{~nL}$ each, then dispensed into the appropriate acidcontaining wells as one injection. On addition of the template and coupling reagent solution, the well contents were mixed three times, with pipette tips moving $1 \mathrm{~mm}$ per well in order to fully mix components. The resulting reaction volumes were each $2 \mu \mathrm{L}$. The 384-well plate was sealed, and the Nano Nest was placed in a heated oven set at $60{ }^{\circ} \mathrm{C}$ for $18 \mathrm{~h}$. Using an Eppendorf multi pipette, the reaction was then quenched with $2 \mu \mathrm{L}$ of a DMSO stock solution; it was further diluted with DMSO to yield a $10 \mu \mathrm{L}$ volume $(20 \mathrm{mM})$. Then $1 \mu \mathrm{L}$ was transferred to a 384-well analytical COC plate containing $19 \mu \mathrm{L}$ of DMSO, for a final volume of $20 \mu \mathrm{L}$ (1 mM solution). The plate was analyzed by ADE-OPI-MS along with UPLC-MS (Agilent 1290/6150). For ADE-OPI-MS detection, $5 \mathrm{~nL}$ samples were injected and analyzed with full mass scan mode (100-1000Da, 100ms accumulation time). Plate mapping of acid reactant (monomer) and expected product molecular weight and molecular formula can be found in Supplemental Table 3. Raw data containing the LC area ratios for the 192-well plate can be found in Supplemental Table 4.

Experiment 3. Amide formation: reaction of DNA headpiece with 96 acids using HATU as coupling agent, as illustrated in Supplemental Figure 6.

On-DNA chemical transformations are typically carried out using a short headpiece DNA $([\mathrm{M}]=$ 4937.23 $\mathrm{Da}$ (purchased as a SEC purified lyophilate from LGC Biosearch Technologies, Petaluma, CA); duplex sequence 5'-/5Phos/GAGTCA/iSp9//iUniAmM//iSp9/TGACTCCC-3') at 10-20 nmol scale. Following on-DNA chemistry, the DNA is precipitated $\left(5 \mathrm{M} \mathrm{NaCl} / \mathrm{EtOH},-20{ }^{\circ} \mathrm{C}\right)$, re-dissolved in water and analyzed by oligonucleotide-LC-UV-MS $(10 \mu \mathrm{M}$ in water; $40-80$ pmol/injection; Xevo G2-XS TOF; negative ESI-MS.

A 384-well plate experiment examining the reactivity of commercially available DNA possessing an amine reaction point was carried out under dehydrating coupling conditions. The reactions were run at 5 nanomolar scale; a Thermo Scientific 12-channel multi pipette (E1 ClipTip 2-125uL model 
$\mathrm{OH} 26467$ ) was used to dose from a 384-well source plate containing stock solutions of starting materials and reagents into a 384-well reaction plate.

Stock solutions of each reaction component were prepared: DNA headpiece as a $1 \mathrm{mM}$ solution in $250 \mathrm{mM}$ trisborate buffer $(\mathrm{pH} 9.5)$ and $200 \mathrm{mM}$ DMAc stock solutions of DIEA, acid monomers, and HATU. A multi pipette was used to dispense $1.25 \mu \mathrm{L}$ (50 equiv.) of the acid monomer solution to the appropriate low-volume well (Labcyte, Cat. No. LP0200) in a 96-well pattern ranging from A1-D24, followed by $1.25 \mu \mathrm{L}$ of DIEA (50 equiv.) and 1.25 $\mu \mathrm{L}$ of HATU (50 equiv.) $200 \mathrm{mM}$ stock solutions. After addition of the HATU solution, the $3.75 \mu \mathrm{L}$ solutions were shaken with an Eppendorf oscillating shaker (400 rpm) for 5 minutes, and then allowed to stand for another 5 minutes at room temperature. On completion of this premixing, the DNA solution $(5 \mu \mathrm{L})$ was dispensed, yielding a final DNA reaction concentration of $0.57 \mathrm{mM}$ in a volume of $8.75 \mu \mathrm{L}$. The plate was sealed with a PFA sheet (Analytical Sales and Services, contact for Cat. No.), shaken for 5 minutes, and subsequently allowed to stand at room temperature for 2 hours.

Using an Eppendorf multi pipette, the reaction was diluted to $20 \mu \mathrm{L}$ with water (Fisher Optima), providing a $0.25 \mathrm{mM}$ DNA solution (in a 4:1 mixture of $62.5 \mathrm{mM}$ trisborate buffer) / DMAc). The plate was analyzed by ADE-OPI-MS followed by UPLC-MS on a Waters TOF instrument. For ADE-OPI-MS detection, 10nL samples were injected and analyzed with full mass scan mode (2002500Da, 150ms accumulation time). Plate mapping of acid reactant (monomer) and expected product molecular and formula weight can be found in Supplemental Table 5. 


\section{Supplemental Figures}

(A) Screen shot of the customized software (Virscidian Inc. Cary, N.C.) for plate-based data processing:

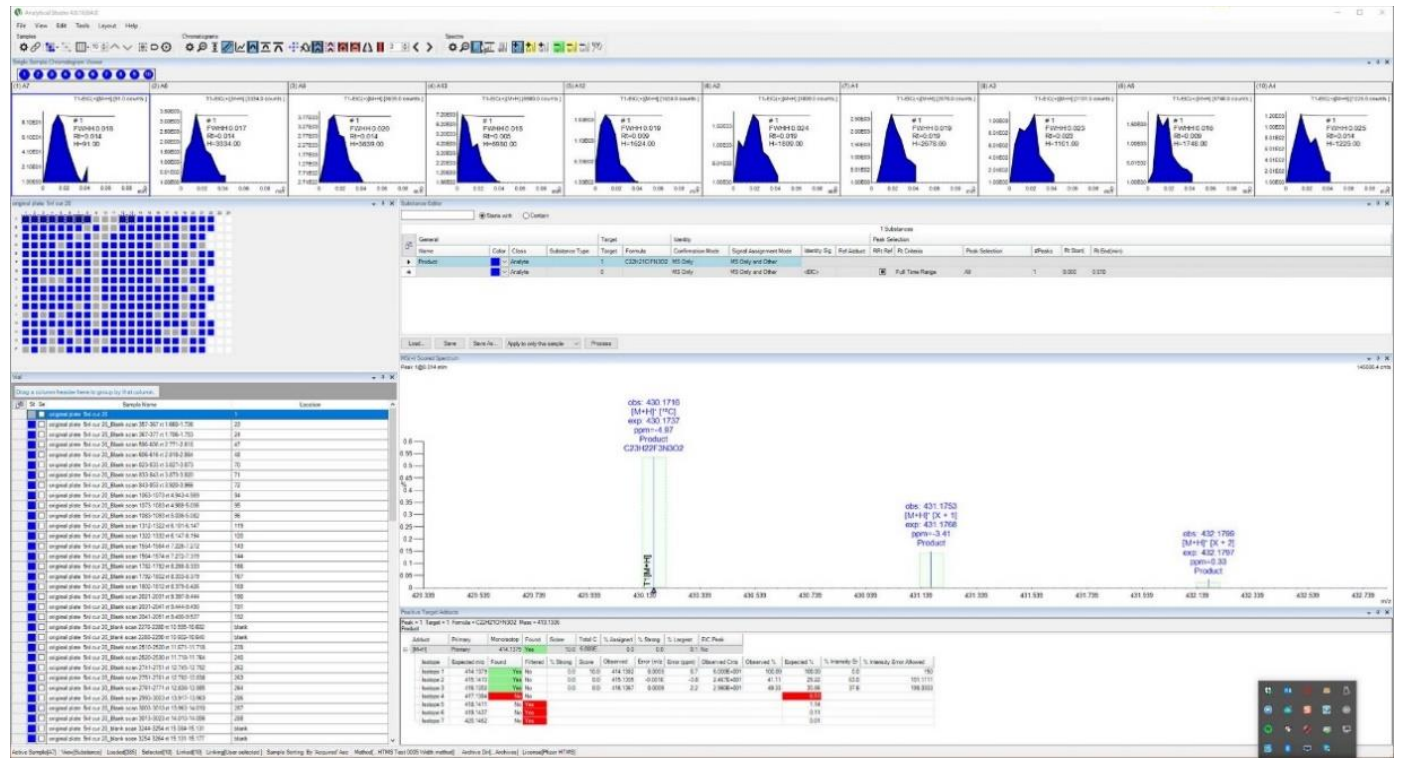

(B) Heatmap of product formation, Experiment 2, ADE-OPI-MS, replicate 1. (C) Heatmap of product formation, replicate 2 .
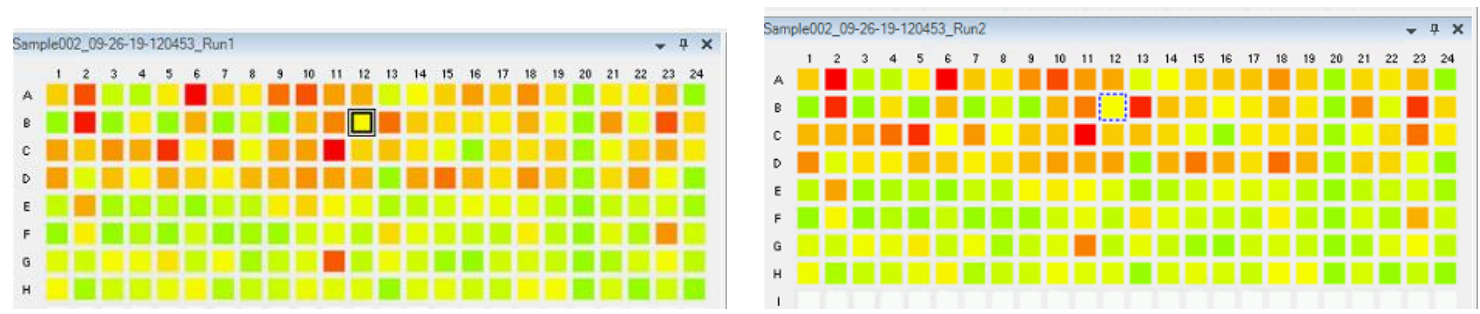

Supplemental Figure 1. Data processing software and product formation for Experiment 2. Within a few minutes, hundreds of chemical reactions can be evaluated with the ADE-OPI-MS platform, providing HRMS data for each reaction. These information-rich datasets require powerful software solutions for data processing, in order to prevent data handling from becoming a new bottleneck. To address this, an algorithm was specifically designed to handle plate-based high-content MS data. Prototype software (Virscidian Inc., Cary, N.C.) is shown (Figure 1A). With a simple input .csv file listing all the pertinent formulas, such as the template, monomer, and targeted product(s), the software can extract and quantify all molecules of interest from each well. Product formation can be displayed in heatmaps regardless of whether the same products are generated in each reaction. A repeated injection of 192 reactions provided a similar heatmap pattern of production (Figure 1B and Figure 1C; the heatmap gradient of red to yellow to green reflects the high to low peak areas of targeted products in each well), highlighting the reproducibility of both the ADE-OPI-MS analytical platform and this data processing software. 
A.
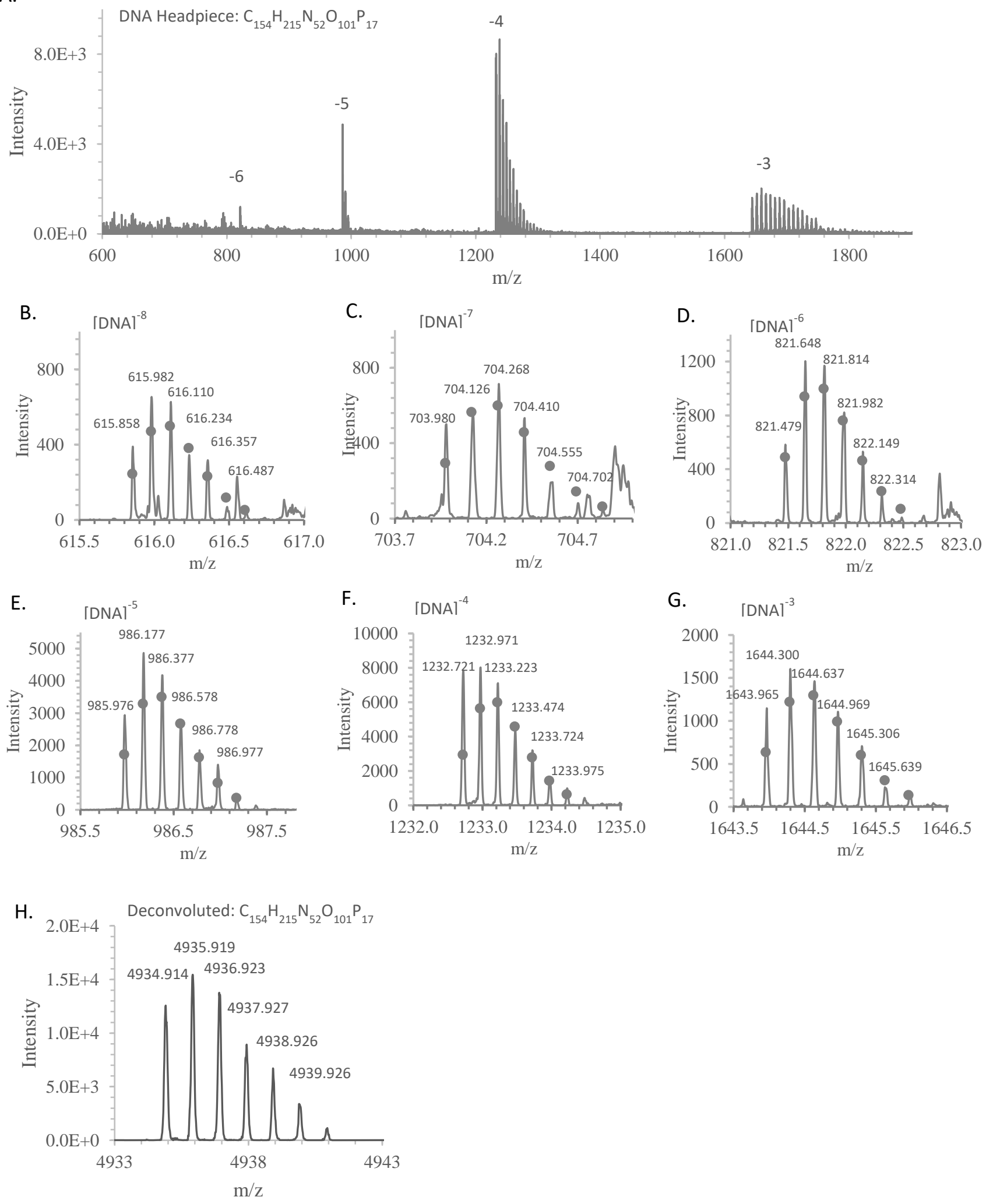

Supplemental Figure 2. Analysis of results from Experiment 3.ADE-OPI-MS works well not only for analysis of small molecules, but also for larger molecules such as precursors to DNA-encoded chemical 
library (DECL) compounds. (A) A DNA headpiece was analyzed directly with ADE-OPI-MS. Multiple charge envelopes were observed, ranging from -3 to -8 . (B)-(G) Zoom-in views of the peaks detected at different charge states: -8 to -3 . (H) Deconvoluted masses for the DNA headpiece, with all of the differently charged ions that were observed. All detected peaks matched well with the calculated values for $m / z$ and relative intensity (shown as solid dots). 
Supplemental Table 1: List of the observed peaks at different charge states for the DNA headpiece.

\begin{tabular}{cccc}
\hline Charge & $m / z$ expected & $m / z$ observed & Accuracy $(\mathrm{ppm})$ \\
\hline-3 & 1643.952 & 1643.965 & 7.9 \\
-4 & 1232.712 & 1232.721 & 7.3 \\
-5 & 985.968 & 985.976 & 8.1 \\
-6 & 821.472 & 821.479 & 8.0 \\
-7 & 703.975 & 703.980 & 7.1 \\
-8 & 615.852 & 615.858 & 9.7 \\
& & Average & 8.0 \\
-1 & 4934.882 & 4934.914 & 6.7 \\
(deconvoluted) & & & \\
\hline
\end{tabular}



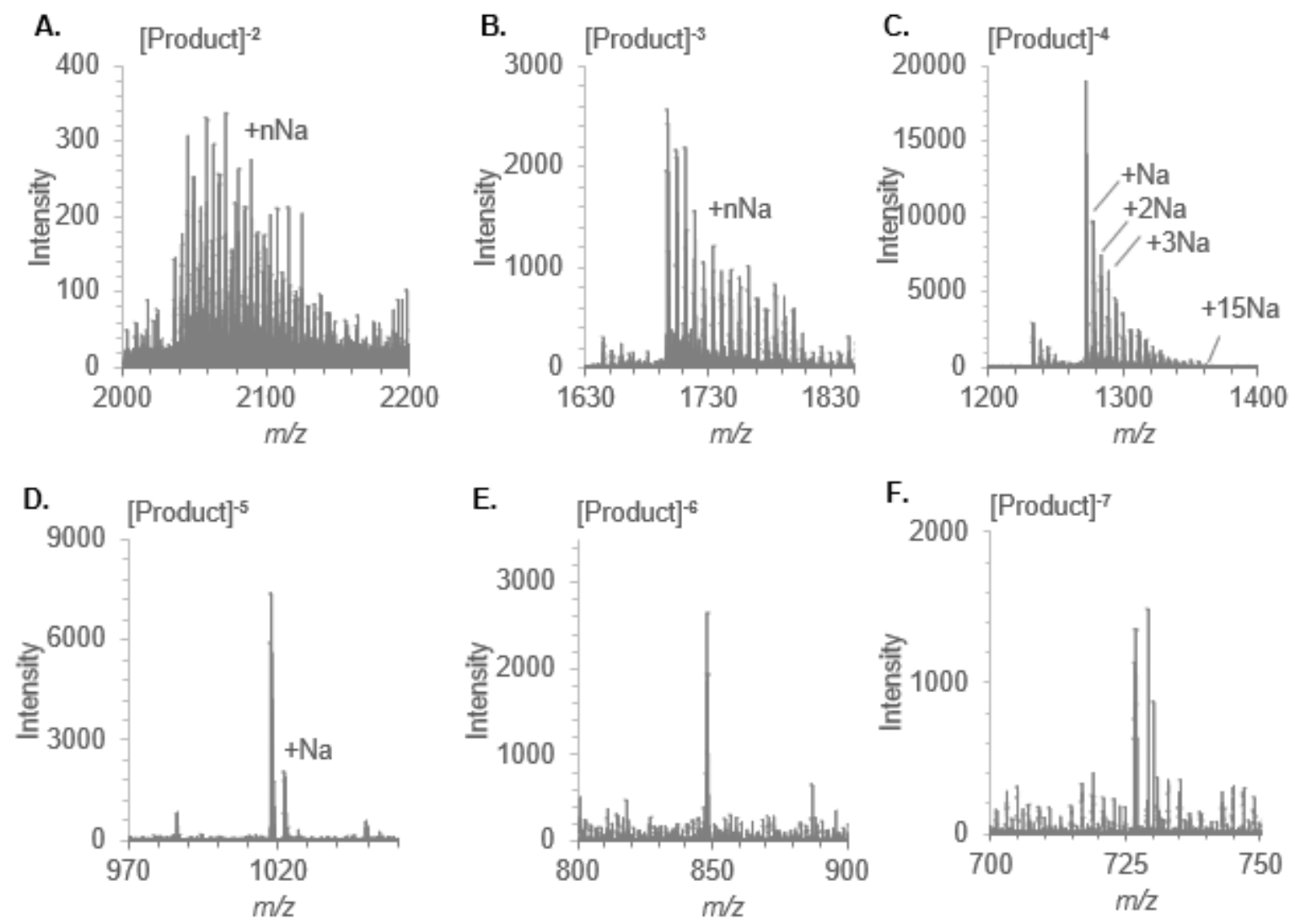

G.

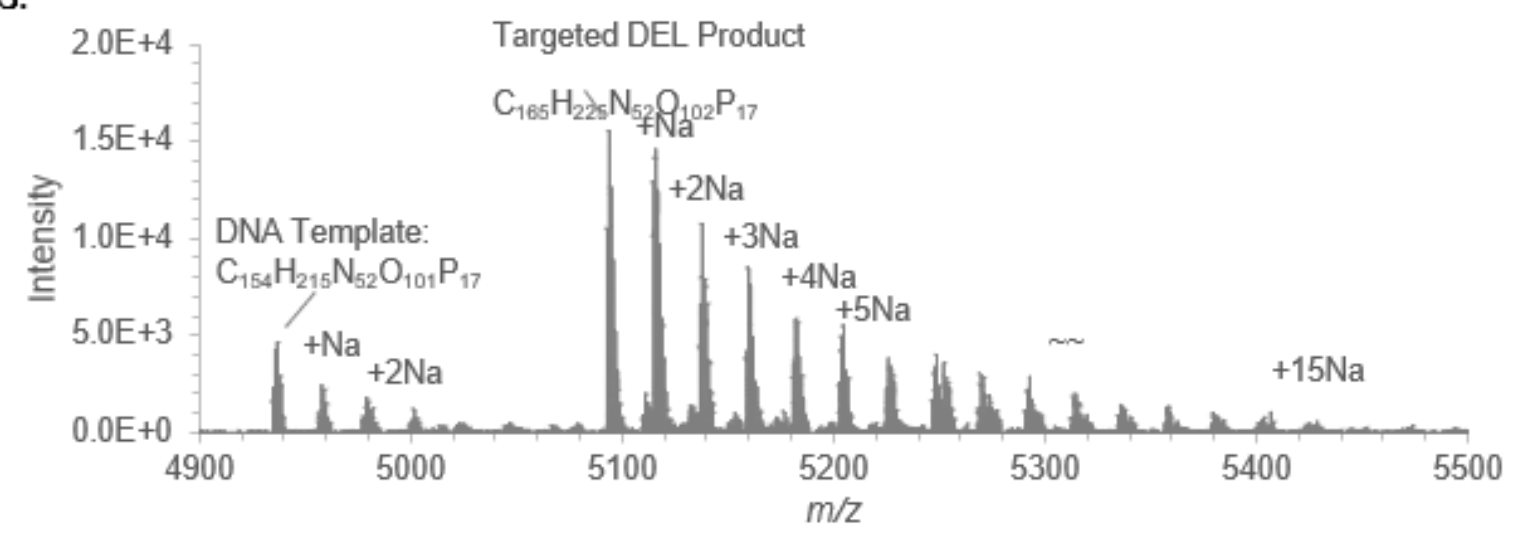

Supplemental Figure 3. Experiment 3: Examination of sample H04.

DNA headpiece-derived compounds can be detected at different charge states, as shown for the product in well $\mathrm{H} 04$ (targeted formula: $\mathrm{C}_{165} \mathrm{H}_{225} \mathrm{~N}_{52} \mathrm{O}_{102} \mathrm{P}_{17}$ ): species with -2 to -7 charges are shown in Figures $3 \mathrm{~A}-$ 3 F. Ions with a -4 charge (Figure $3 \mathrm{C}$ ) display the highest MS signal, followed by $-5,-3$, and -6 . Multiple $\mathrm{Na}$ adducts were also observed, especially for ions with a charge of -2 to -4 (Figures 3A-3C). Figure 3G depicts the multiple $\mathrm{Na}$ adducts observed upon deconvolution of the raw data to singly charged peaks: more than $10 \mathrm{Na}$ adducts could be observed, particularly for the $-4,-3$, and -2 ions. These Na adduct cluster peaks make the interpretation of spectra difficult. Ions with -5 or more charges possess significantly fewer 
$\mathrm{Na}$ adducts (Figure 3D), while ions with a -6 charge suffered from decreased sensitivity (Figure 3E); the DNA template peaks for these ions were not distinguishable. Ions with a -5 charge are good candidates for qualitative peak identification, as they offer a balance of sensitivity and spectral complexity. 
A.

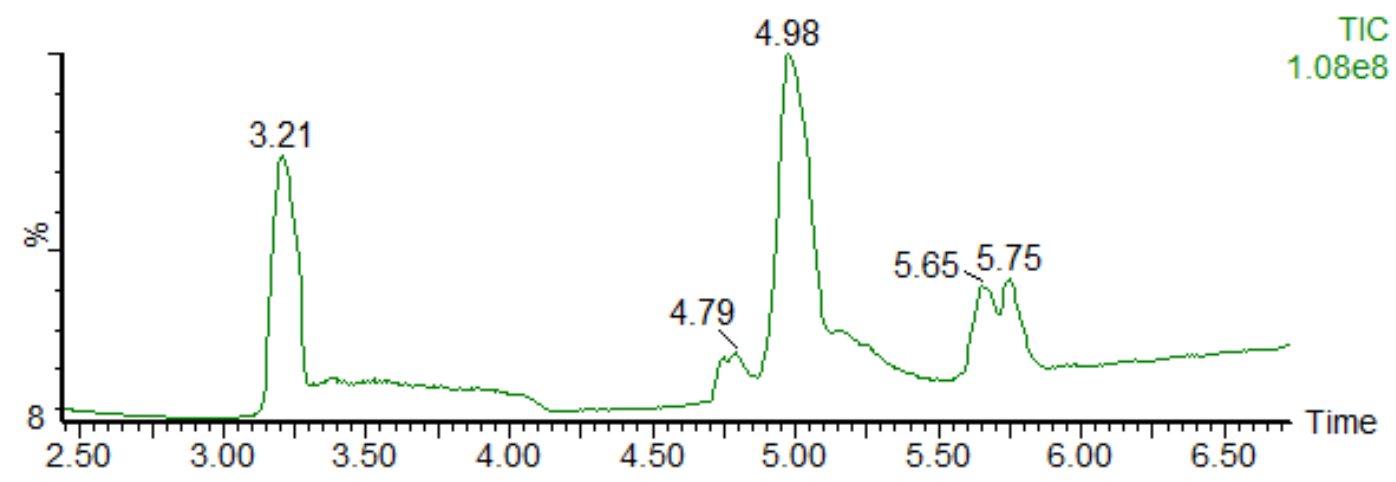

B.
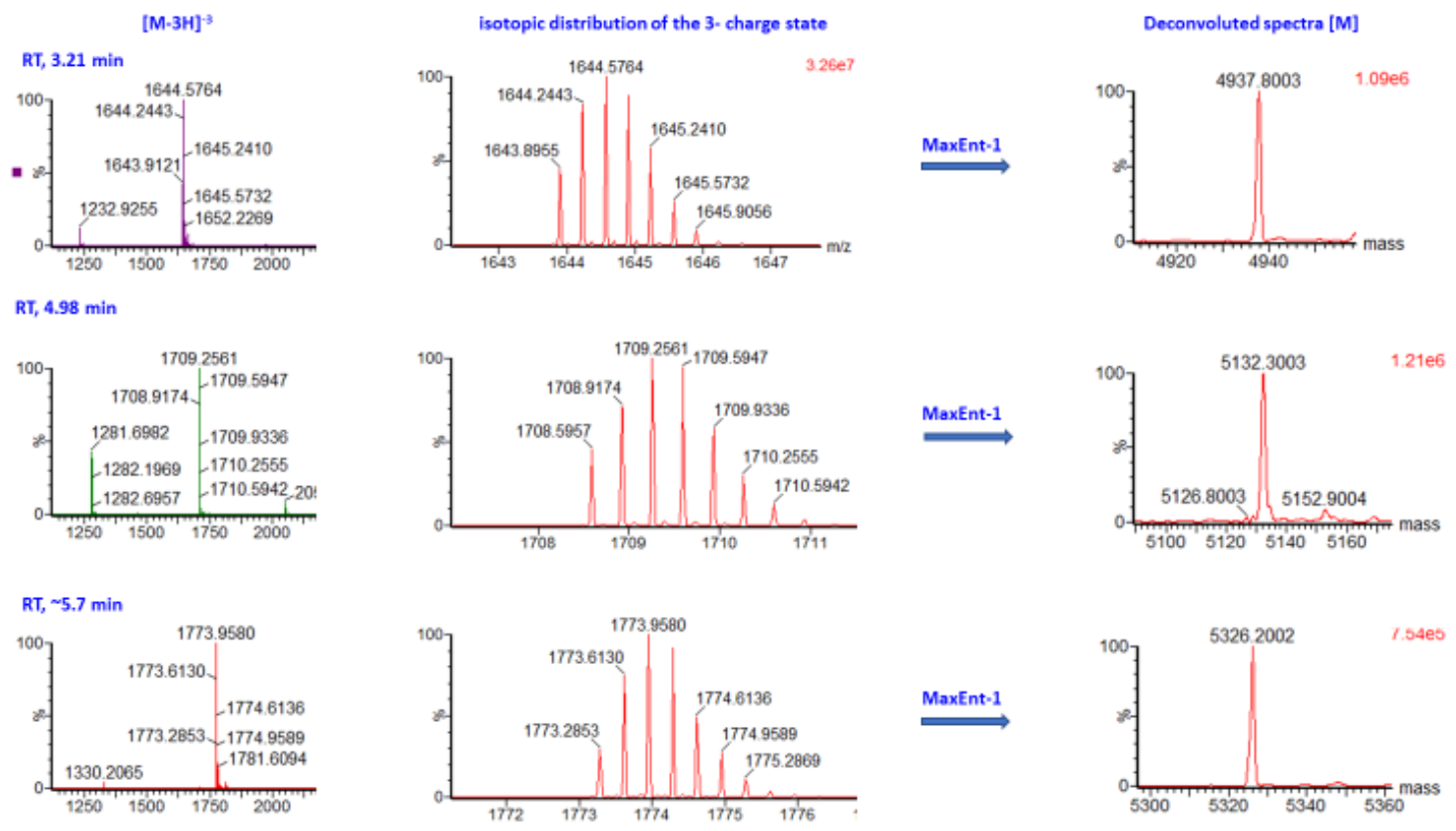

Supplemental Figure 4. Experiment 3: Waters MS-TOF data deconvolution with MaxEnt-1, from LC/UV method.

(A) DNA headpiece-derived compound from well A02 (A2): TIC as acquired in Profile mode on a Waters MS-TOF with starting DNA headpiece at $3.21 \mathrm{~min}$, expected product at $4.98 \mathrm{~min}$, and unknown byproducts at 5.65-5.75 min. (B) $[\mathrm{M}-3 \mathrm{H}]^{-3}$ is the most abundant charge state for these DNA compounds. Expansion of the charge cone provides the isotopic distribution, which can be used to derive deconvoluted spectra of detected compounds through use of MaxEnt. 
Supplemental Table 2. Raw UPLC-MS data used for visual plots

Experiment 1: C-N Pd reaction optimization screen

\begin{tabular}{|c|c|c|c|c|c|}
\hline $\begin{array}{l}\text { Well/sample } \\
\text { No. }\end{array}$ & Location & Base & Catalyst & $\begin{array}{l}\text { Relative product } \\
\text { conversion * }\end{array}$ & $\begin{array}{l}\text { OPI/MS } \\
\text { Area }\end{array}$ \\
\hline 1 & A1 & DBU & DPPF G3 & 0 & 0 \\
\hline 2 & A2 & MTBD & DPPF G3 & 0 & 0 \\
\hline 3 & A3 & BTMG & DPPF G3 & 0 & 0 \\
\hline 4 & A4 & BEMP & DPPF G3 & 0 & 0 \\
\hline 5 & A5 & BTTP & DPPF G3 & 0 & 0 \\
\hline 6 & A6 & P2-Et & DPPF G3 & 0 & 0 \\
\hline 7 & B1 & DBU & Xantphos G3 & 0 & 0 \\
\hline 8 & B2 & MTBD & Xantphos G3 & 0 & 7262 \\
\hline 9 & B3 & BTMG & Xantphos G3 & 0 & 23530 \\
\hline 10 & B4 & BEMP & Xantphos G3 & 0 & 31390 \\
\hline 11 & B5 & BTTP & Xantphos G3 & 0 & 14680 \\
\hline 12 & B6 & P2-Et & Xantphos G3 & 0 & 4863 \\
\hline 13 & $\mathrm{C} 1$ & DBU & RuPhos G3 & 0 & 0 \\
\hline 14 & $\mathrm{C} 2$ & MTBD & RuPhos G3 & 0 & 39340 \\
\hline 15 & $\mathrm{C} 3$ & BTMG & RuPhos G3 & 3.35500 & 75680 \\
\hline 16 & $\mathrm{C} 4$ & BEMP & RuPhos G3 & 0 & 94630 \\
\hline 17 & $\mathrm{C} 5$ & BTTP & RuPhos G3 & 5.54901 & 122000 \\
\hline 18 & C6 & P2-Et & RuPhos G3 & 7.90067 & 208700 \\
\hline 19 & D1 & $\overline{\mathrm{DBU}}$ & $\begin{array}{l}\text { t-butylXPhos } \\
\text { G3 }\end{array}$ & 0 & 90770 \\
\hline 20 & D2 & MTBD & $\begin{array}{l}\text { t-butylXPhos } \\
\text { G3 }\end{array}$ & 44.27896 & 630600 \\
\hline 21 & D3 & BTMG & $\begin{array}{l}\text { t-butylXPhos } \\
\text { G3 }\end{array}$ & 24.20925 & 555400 \\
\hline 22 & D4 & BEMP & $\begin{array}{l}\text { t-butylXPhos } \\
\text { G3 }\end{array}$ & 7.93125 & 200700 \\
\hline 23 & D5 & BTTP & $\begin{array}{l}\text { t-butylXPhos } \\
\text { G3 }\end{array}$ & 8.90609 & 198100 \\
\hline 24 & D6 & P2-Et & $\begin{array}{l}\text { t-butylXPhos } \\
\text { G3 }\end{array}$ & 29.22655 & 626500 \\
\hline 25 & E1 & DBU & $\begin{array}{l}\text { AdBrettPhos } \\
\text { G3 }\end{array}$ & 0 & 0 \\
\hline 26 & E2 & MTBD & $\begin{array}{l}\text { AdBrettPhos } \\
\text { G3 }\end{array}$ & 0 & 7329 \\
\hline 27 & E3 & BTMG & $\begin{array}{l}\text { AdBrettPhos } \\
\text { G3 }\end{array}$ & 0 & 6445 \\
\hline 28 & $\mathrm{E} 4$ & BEMP & $\begin{array}{l}\text { AdBrettPhos } \\
\text { G3 }\end{array}$ & 0 & 3546 \\
\hline
\end{tabular}




\begin{tabular}{|c|c|c|c|c|c|}
\hline 29 & E5 & BTTP & $\begin{array}{l}\text { AdBrettPhos } \\
\text { G3 }\end{array}$ & 0 & 4838 \\
\hline 30 & E6 & P2-Et & $\begin{array}{l}\text { AdBrettPhos } \\
\text { G3 }\end{array}$ & 0 & 13780 \\
\hline 31 & F1 & $\overline{\mathrm{DBU}}$ & $\begin{array}{l}\text { tbuBrettPhos } \\
\text { Pd G3 }\end{array}$ & 0 & 0 \\
\hline 32 & $\mathrm{~F} 2$ & MTBD & $\begin{array}{l}\text { tbuBrettPhos } \\
\text { Pd G3 }\end{array}$ & 0 & 9534 \\
\hline 33 & F3 & BTMG & $\begin{array}{l}\text { tbuBrettPhos } \\
\text { Pd G3 }\end{array}$ & 0 & 11200 \\
\hline 34 & F4 & BEMP & $\begin{array}{l}\text { tbuBrettPhos } \\
\text { Pd G3 }\end{array}$ & 0 & 3451 \\
\hline 35 & F5 & BTTP & $\begin{array}{l}\text { tbuBrettPhos } \\
\text { Pd G3 }\end{array}$ & 0 & 14380 \\
\hline 36 & F6 & P2-Et & $\begin{array}{l}\text { tbuBrettPhos } \\
\text { Pd G3 }\end{array}$ & 0 & 31910 \\
\hline 37 & G1 & DBU & BrettPhos G3 & 0 & 0 \\
\hline 38 & G2 & MTBD & BrettPhos G3 & 0 & 0 \\
\hline 39 & G3 & BTMG & BrettPhos G3 & 0 & 0 \\
\hline 40 & G4 & BEMP & BrettPhos G3 & 0 & 1801 \\
\hline 41 & G5 & BTTP & BrettPhos G3 & 0 & 3143 \\
\hline 42 & G6 & P2-Et & BrettPhos G3 & 4.57673 & 144600 \\
\hline 43 & $\mathrm{H} 1$ & DBU & BINAP G3 & 0 & 0 \\
\hline 44 & $\mathrm{H} 2$ & MTBD & BINAP G3 & 0 & 0 \\
\hline 45 & $\mathrm{H} 3$ & BTMG & BINAP G3 & 0 & 0 \\
\hline 46 & $\mathrm{H} 4$ & BEMP & BINAP G3 & 0 & 0 \\
\hline 47 & H5 & BTTP & BINAP G3 & 0 & 0 \\
\hline 48 & H6 & $\mathrm{P} 2-\mathrm{Et}$ & BINAP G3 & 0 & 4807 \\
\hline 49 & I1 & DBU & tBu3P G3 & 0 & 0 \\
\hline 50 & I2 & MTBD & tBu3P G3 & 0 & 1815 \\
\hline 51 & I3 & BTMG & tBu3P G3 & 0 & 10920 \\
\hline 52 & I4 & BEMP & tBu3P G3 & 0 & 13890 \\
\hline 53 & I5 & BTTP & tBu3P G3 & 0 & 50930 \\
\hline 54 & I6 & P2-Et & tBu3P G3 & 0 & 11800 \\
\hline 55 & $\mathrm{~J} 1$ & $\overline{\mathrm{DBU}}$ & $\begin{array}{l}\text { PPA } \\
\text { (MeCgPPh) G3 }\end{array}$ & 0 & 0 \\
\hline 56 & $\mathrm{~J} 2$ & MTBD & $\begin{array}{l}\text { PPA } \\
(\mathrm{MeCgPPh}) \mathrm{G} 3\end{array}$ & 0 & 0 \\
\hline 57 & J3 & BTMG & $\begin{array}{l}\text { PPA } \\
(\mathrm{MeCgPPh}) \mathrm{G} 3\end{array}$ & 0 & 0 \\
\hline 58 & $\mathrm{~J} 4$ & BEMP & $\begin{array}{l}\text { PPA } \\
(\mathrm{MeCgPPh}) \mathrm{G} 3\end{array}$ & 0 & 0 \\
\hline 59 & J5 & BTTP & $\begin{array}{l}\text { PPA } \\
\text { (MeCgPPh) G3 }\end{array}$ & 0 & 0 \\
\hline
\end{tabular}




\begin{tabular}{|c|c|c|c|c|c|}
\hline 60 & J6 & P2-Et & $\begin{array}{l}\text { PPA } \\
\text { (MeCgPPh) G3 }\end{array}$ & 0 & 0 \\
\hline 61 & K1 & DBU & Aphos G3 & 0 & 0 \\
\hline 62 & K2 & MTBD & Aphos G3 & 0 & 0 \\
\hline 63 & K3 & BTMG & Aphos G3 & 0 & 0 \\
\hline 64 & K4 & BEMP & Aphos G3 & 0 & 0 \\
\hline 65 & K5 & BTTP & Aphos G3 & 0 & 0 \\
\hline 66 & K6 & $\mathrm{P} 2-\mathrm{Et}$ & Aphos G3 & 0 & 0 \\
\hline 67 & L1 & DBU & Xphos G3 & 0 & 0 \\
\hline 68 & L2 & MTBD & Xphos G3 & 0 & 5889 \\
\hline 69 & L3 & BTMG & Xphos G3 & 0 & 25250 \\
\hline 70 & L4 & BEMP & Xphos G3 & 0 & 5874 \\
\hline 71 & L5 & BTTP & Xphos G3 & 0 & 14240 \\
\hline 72 & L6 & P2-Et & Xphos G3 & 10.26829 & 236900 \\
\hline 73 & M1 & DBU & DTBTF G3 & 0 & 0 \\
\hline 74 & M2 & MTBD & DTBTF G3 & 0 & 0 \\
\hline 75 & M3 & BTMG & DTBTF G3 & 0 & 0 \\
\hline 76 & M4 & BEMP & DTBTF G3 & 0 & 0 \\
\hline 77 & M5 & BTTP & DTBTF G3 & 0 & 0 \\
\hline 78 & M6 & $\mathrm{P} 2-\mathrm{Et}$ & DTBTF G3 & 7.60676 & 199100 \\
\hline 79 & N1 & DBU & Josi G3 & 0 & 0 \\
\hline 80 & $\mathrm{~N} 2$ & MTBD & Josi G3 & 0 & 0 \\
\hline 81 & N3 & BTMG & Josi G3 & 0 & 0 \\
\hline 82 & $\mathrm{~N} 4$ & BEMP & Josi G3 & 0 & 0 \\
\hline 83 & N5 & BTTP & Josi G3 & 0 & 0 \\
\hline 84 & N6 & P2-Et & Josi G3 & 0 & 8482 \\
\hline 85 & $\mathrm{O} 1$ & DBU & Mordal G3 & 0 & 0 \\
\hline 86 & $\mathrm{O} 2$ & MTBD & Mordal G3 & 0 & 21810 \\
\hline 87 & $\mathrm{O} 3$ & BTMG & Mordal G3 & 0 & 29500 \\
\hline 88 & $\mathrm{O} 4$ & BEMP & Mordal G3 & 0 & 12820 \\
\hline 89 & O5 & BTTP & Mordal G3 & 0 & 16920 \\
\hline 90 & O6 & P2-Et & Mordal G3 & 5.67213 & 115200 \\
\hline 91 & P1 & DBU & Rockphos G3 & 0 & 0 \\
\hline 92 & P2 & MTBD & Rockphos G3 & 0 & 0 \\
\hline 93 & P3 & BTMG & Rockphos G3 & 0 & 18340 \\
\hline 94 & P4 & BEMP & Rockphos G3 & 0 & 11600 \\
\hline 95 & P5 & BTTP & Rockphos G3 & 0 & 24080 \\
\hline 96 & P6 & $\mathrm{P} 2-\mathrm{Et}$ & Rockphos G3 & 0 & 74080 \\
\hline
\end{tabular}

*: UV254 product AreaAbs/Int. Std. 


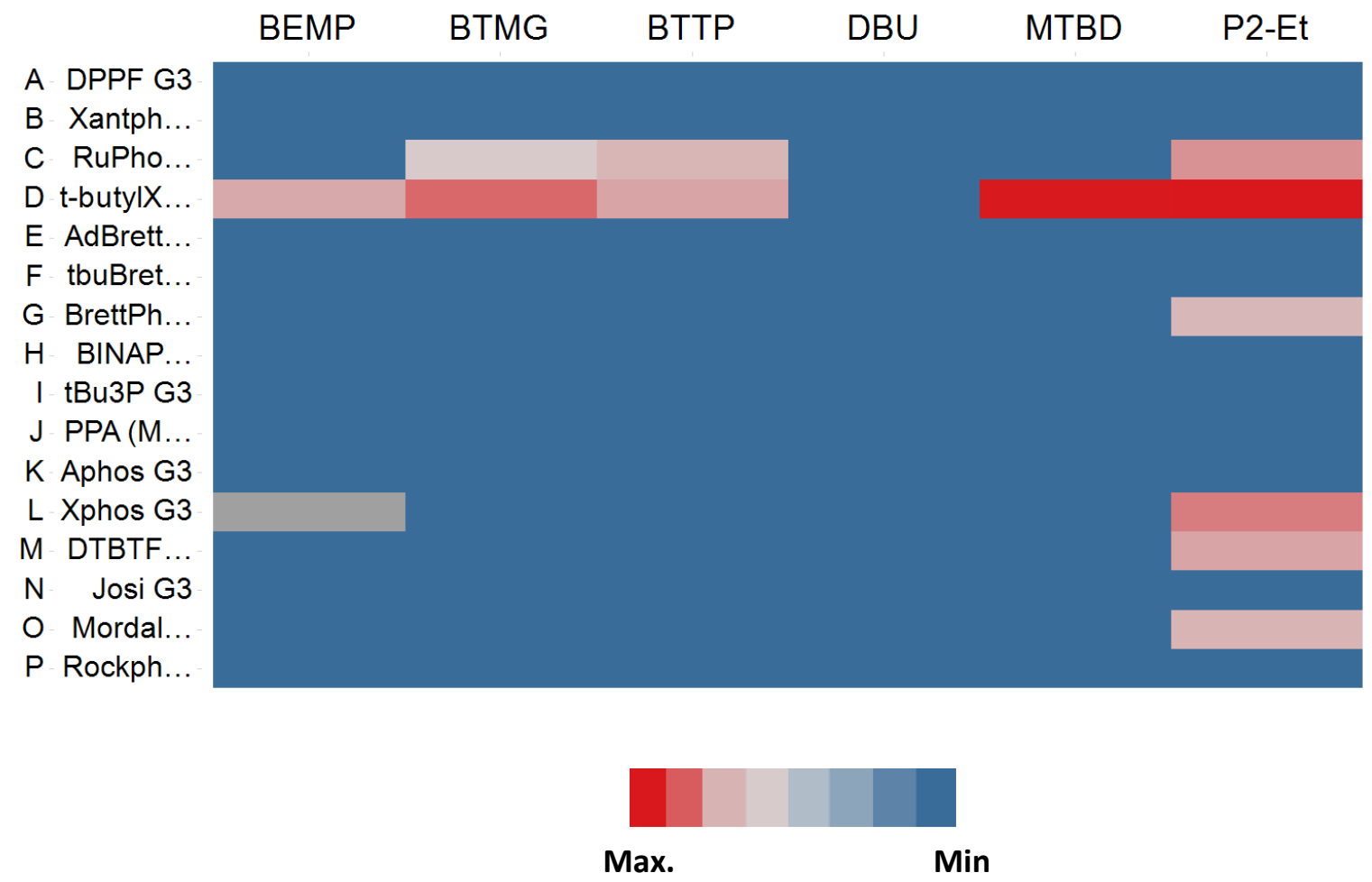

Supplemental Figure 5: Experiment 1 Plate mapping of raw UPLC-MS data 
Experiment 2. Small-molecule amidation reaction
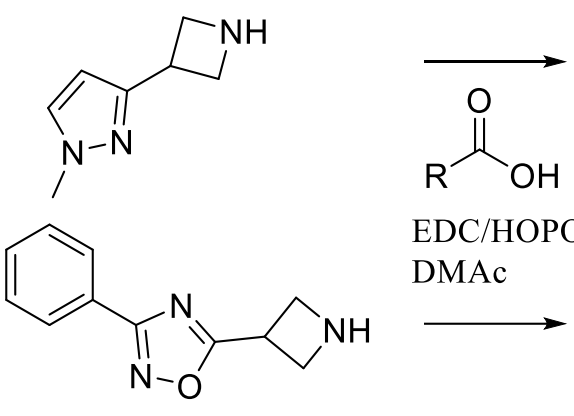<smiles>[R]C(=O)N1CC(c2ccn(C)n2)C1</smiles>

Template 1 A1-D24

EDC/HOPO

DMAc

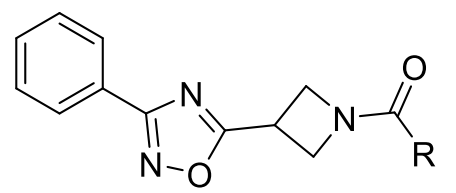

Template 2 E1-H24

Experiment 3. DNA headpiece amidation reaction

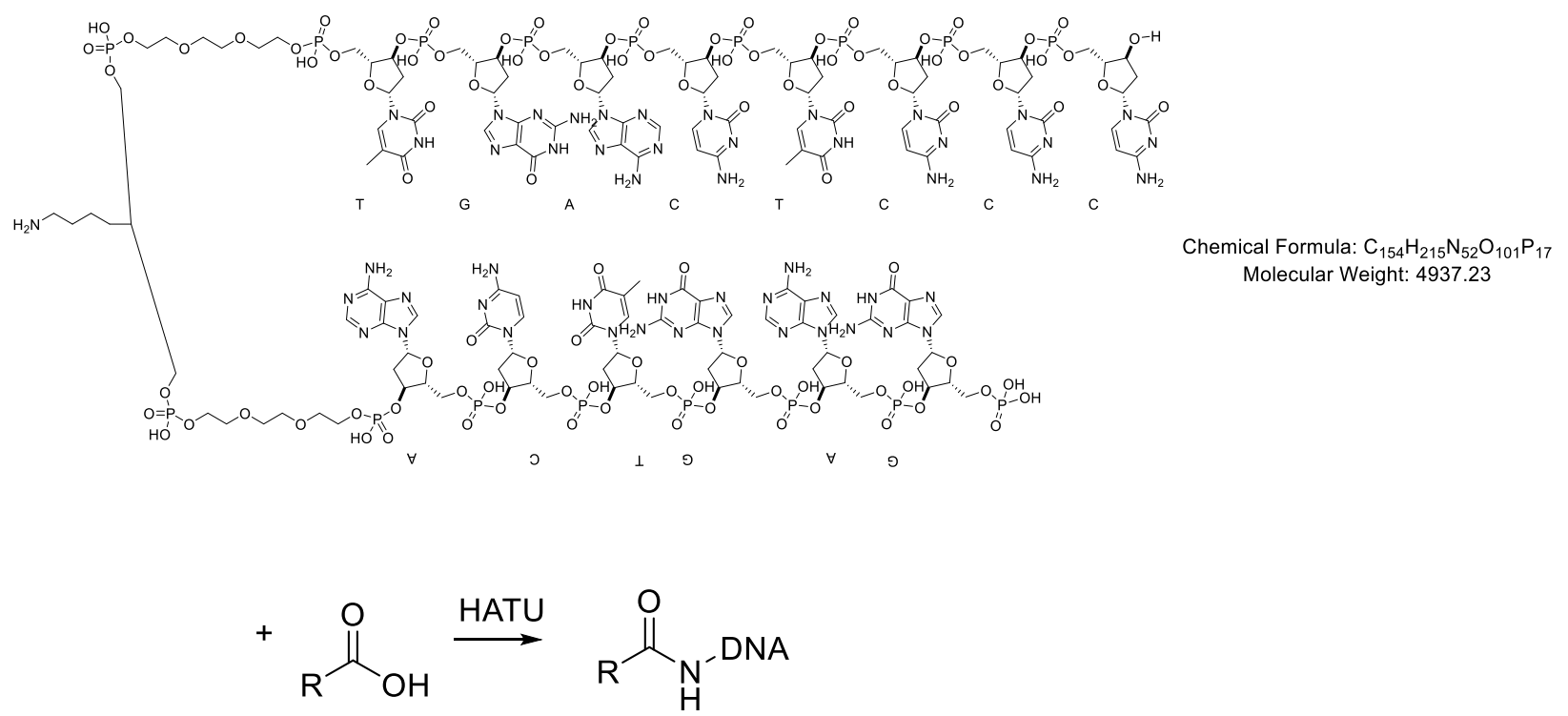

Supplemental Figure 6: Reaction scheme for Experiments 2 and 3.Supplemental Table 3.

Plate mapping of acid reactant and expected product molecular weight and molecular formula for Experiment 2 
Experiment 2. Reaction scheme<smiles>[R]C(=O)OC([R])C1CC(c2ccn(C)n2)CN1C([R])=O</smiles>

$\begin{array}{llc} & \text { Molecular } & \\ \text { Template 1 } & \text { formula } & \text { MW parent } \\ \text { PF-04780968 } & \mathrm{C}_{7} \mathrm{H}_{11} \mathrm{~N}_{3} & 137.18\end{array}$

\begin{tabular}{|c|c|c|c|c|c|}
\hline Location & Product MW & Product MF & Monomer & Monomer MW & Monomer MF \\
\hline A1 & 377.4363 & $\mathrm{C} 22 \mathrm{H} 23 \mathrm{~N} 3 \mathrm{O} 3$ & & 258.28 & C15H14O4 \\
\hline
\end{tabular}




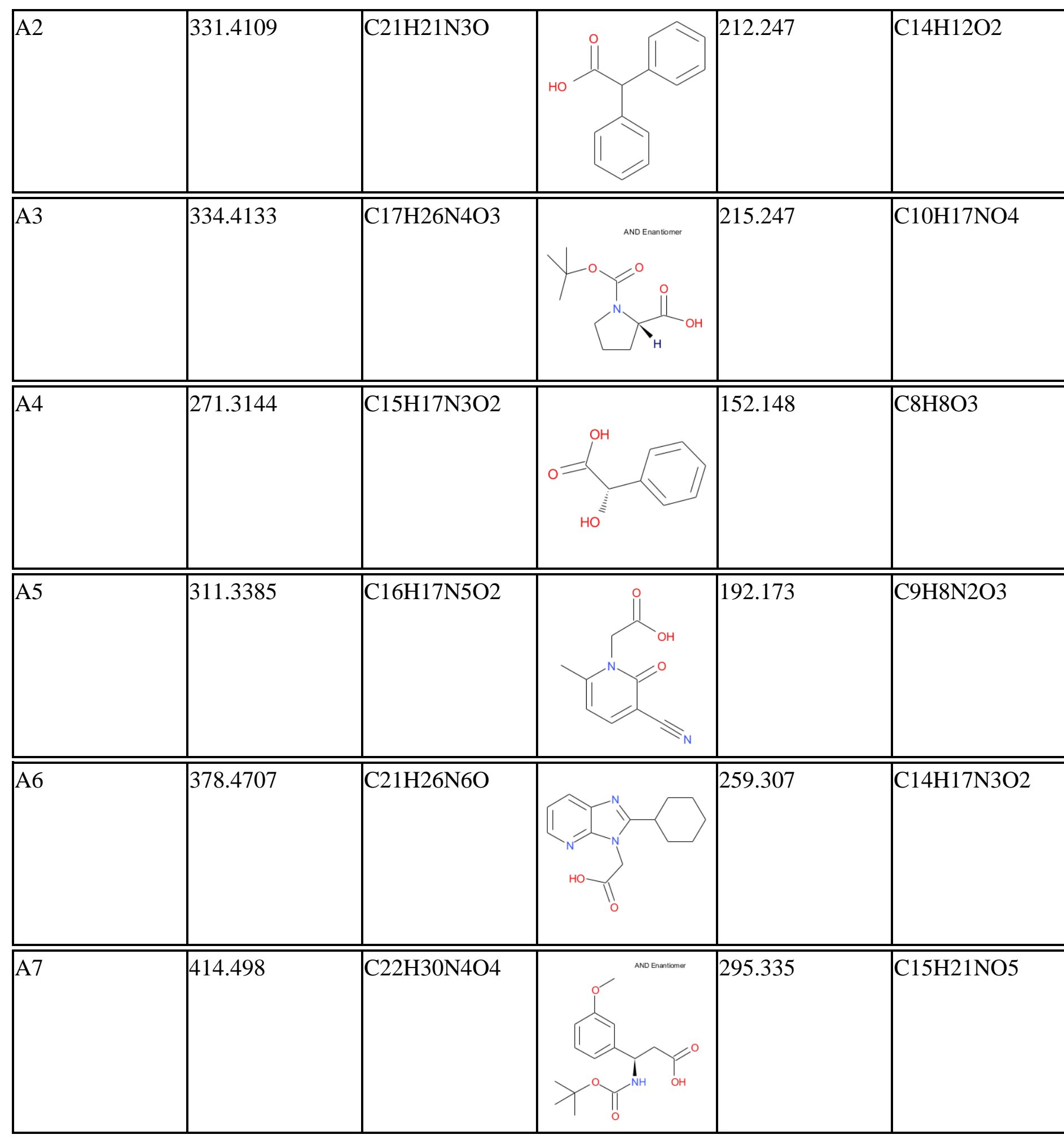




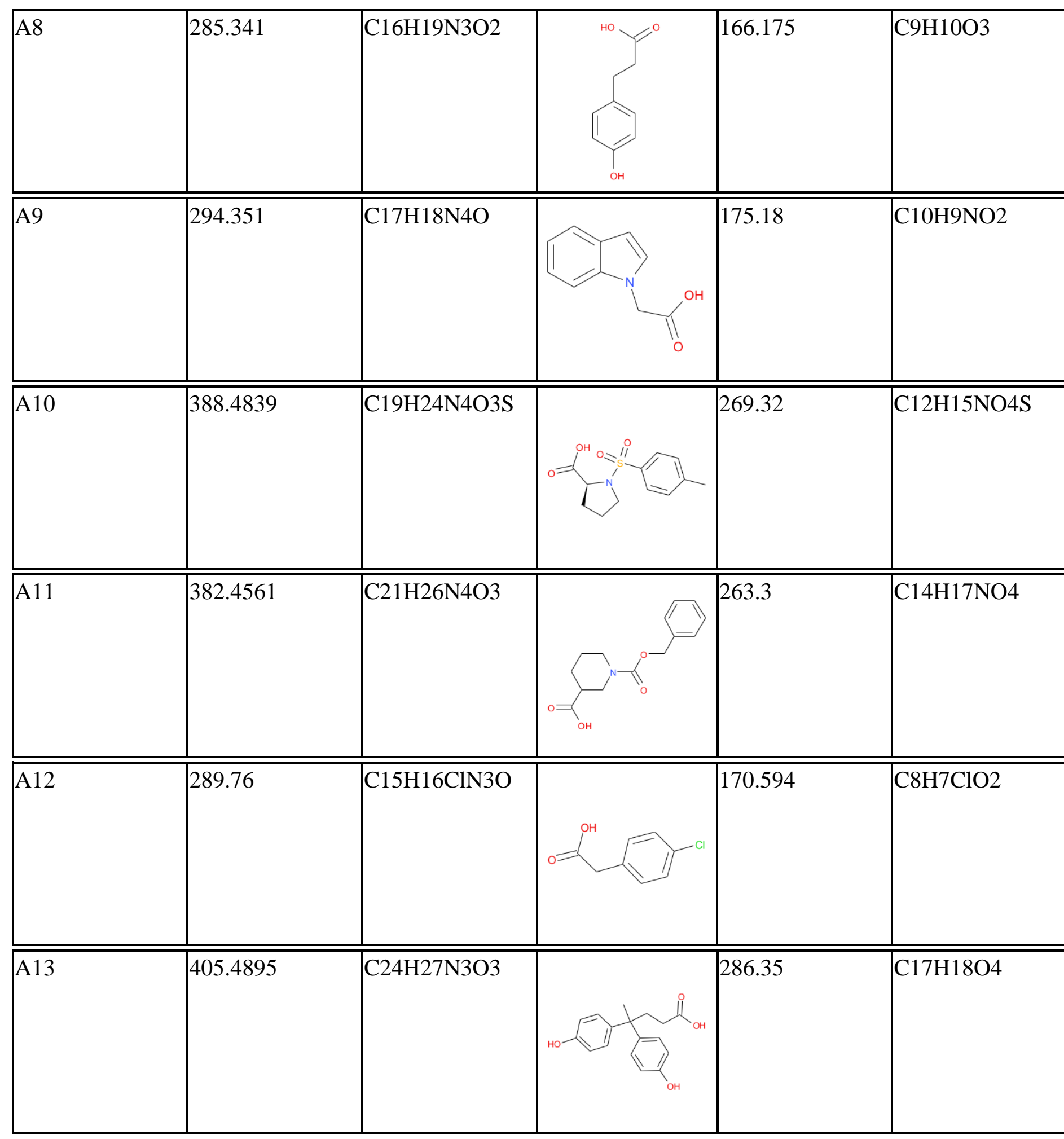




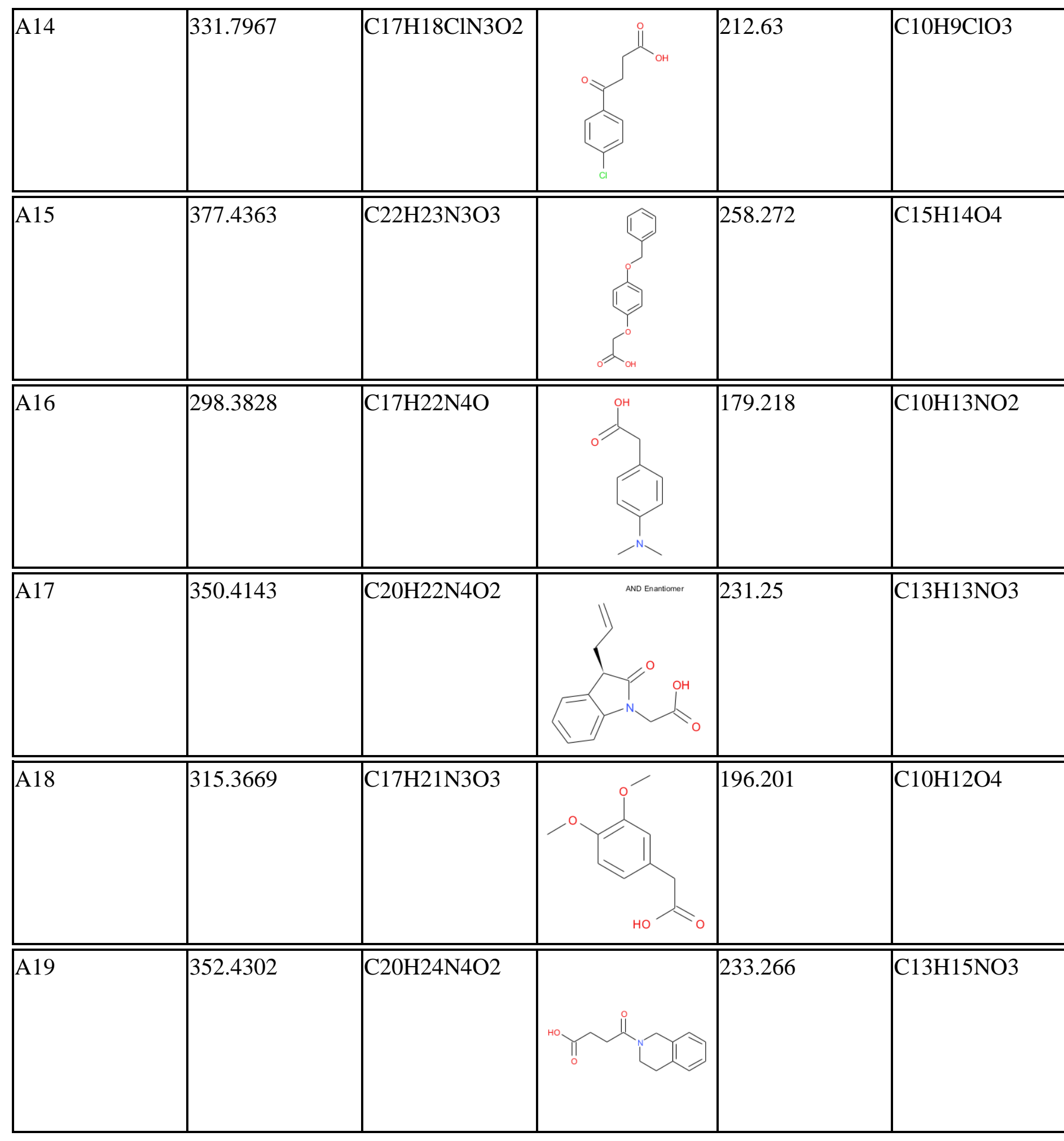




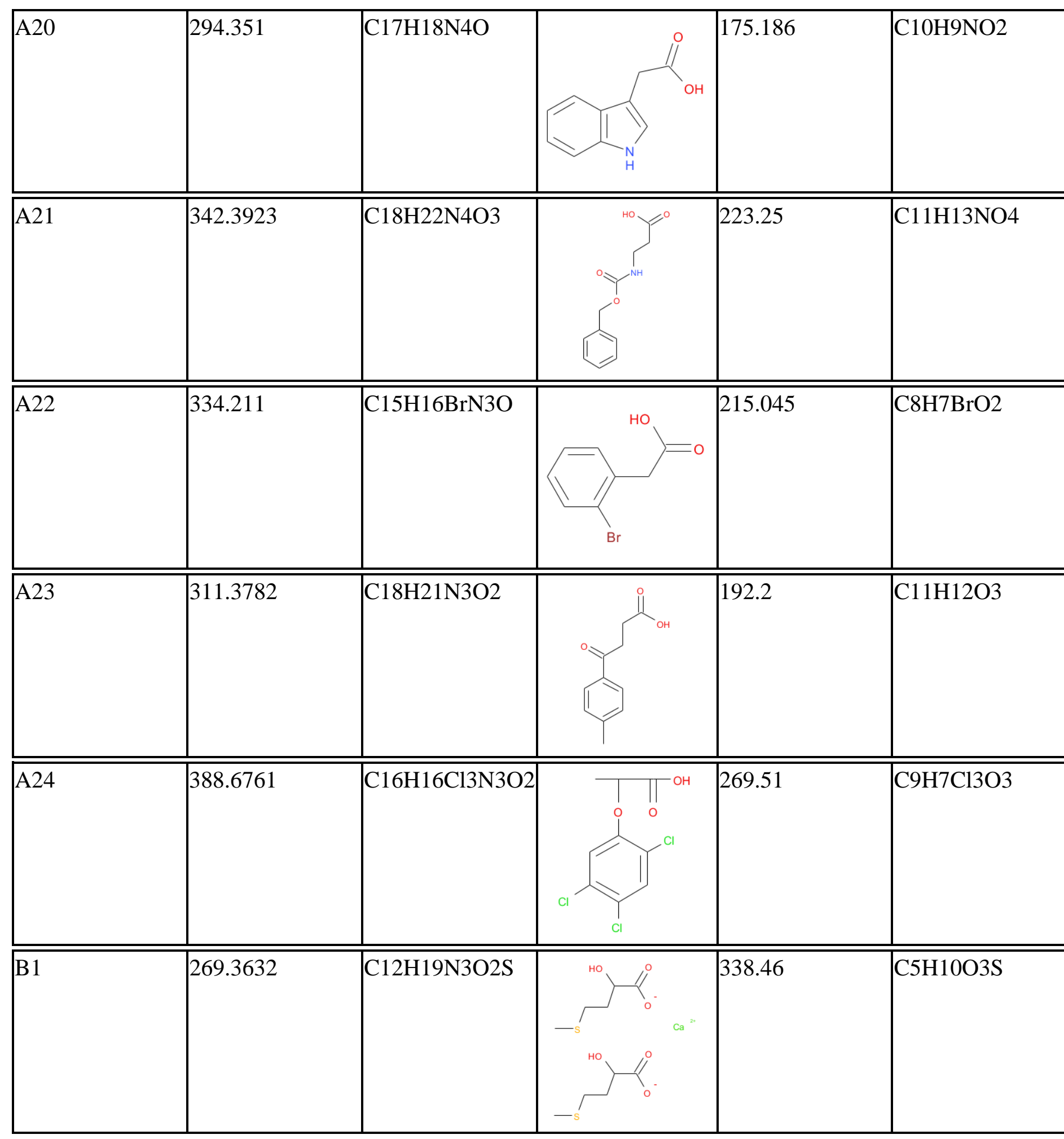




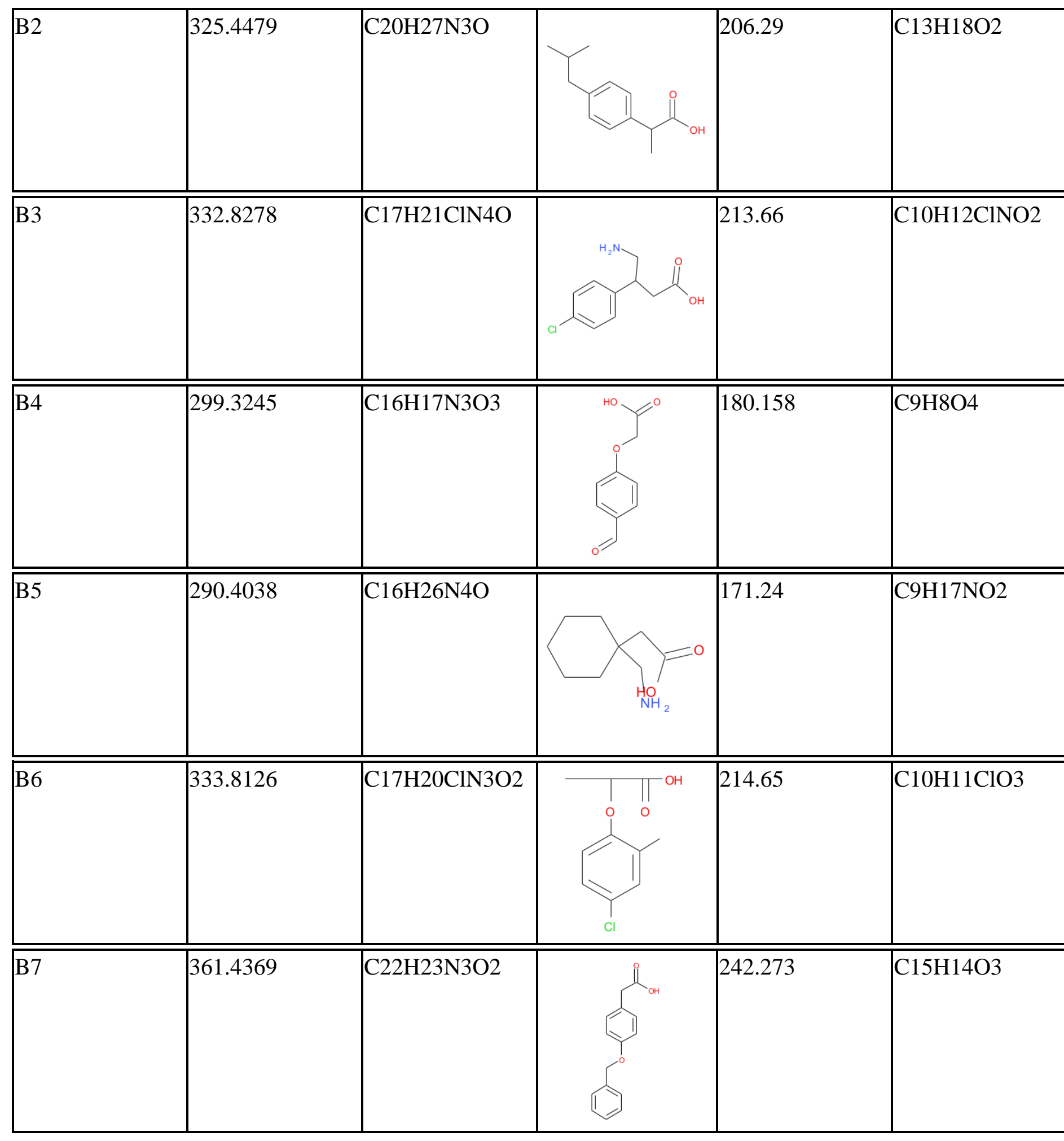




\begin{tabular}{|c|c|c|c|c|}
\hline B8 & 348.4399 & $\mathrm{C} 18 \mathrm{H} 28 \mathrm{~N} 4 \mathrm{O} 3$ & 229.3 & C11H19NO4 \\
\hline B9 & 357.3672 & C16H19N7O3 & 238.202 & C9H10N4O4 \\
\hline B10 & 395.882 & $\mathrm{C} 22 \mathrm{H} 22 \mathrm{ClN} 3 \mathrm{O} 2$ & 276.718 & $\mathrm{C} 15 \mathrm{H} 13 \mathrm{ClO} 3$ \\
\hline B11 & 311.3782 & $\mathrm{C} 18 \mathrm{H} 21 \mathrm{~N} 3 \mathrm{O} 2$ & 192.214 & $\mathrm{C} 11 \mathrm{H} 12 \mathrm{O} 3$ \\
\hline B12 & 298.3397 & C16H18N4O2 & 179.174 & C9H9NO3 \\
\hline B13 & 269.3416 & C16H19N3O & 150.176 & C9H10O2 \\
\hline
\end{tabular}




\begin{tabular}{|c|c|c|c|c|c|}
\hline B14 & 290.3177 & C14H18N4O3 & но $=0$ & 171.151 & C7H9NO4 \\
\hline B15 & 366.3377 & $\mathrm{C} 17 \mathrm{H} 17 \mathrm{~F} 3 \mathrm{~N} 4 \mathrm{O} 2$ & & 247.172 & C10H8F3NO3 \\
\hline B16 & 371.8208 & $\mathrm{C} 18 \mathrm{H} 18 \mathrm{ClN} 5 \mathrm{O} 2$ & & 252.656 & $\mathrm{C} 11 \mathrm{H} 9 \mathrm{ClN} 2 \mathrm{O} 3$ \\
\hline B17 & 285.341 & C16H19N3O2 & & 166.175 & С9H10O3 \\
\hline B18 & 275.3461 & $\mathrm{C} 15 \mathrm{H} 21 \mathrm{~N} 3 \mathrm{O} 2$ & & 156.18 & C8H12O3 \\
\hline B19 & 299.3675 & $\mathrm{C} 17 \mathrm{H} 21 \mathrm{~N} 3 \mathrm{O} 2$ & & 180.202 & $\mathrm{C} 10 \mathrm{H} 12 \mathrm{O} 3$ \\
\hline
\end{tabular}




\begin{tabular}{|c|c|c|c|c|}
\hline B20 & 336.4292 & $\mathrm{C} 17 \mathrm{H} 28 \mathrm{~N} 4 \mathrm{O} 3$ & 217.267 & C10H19NO4 \\
\hline B21 & 297.3517 & C17H19N3O2 & 178.19 & $\mathrm{C} 10 \mathrm{H} 10 \mathrm{O} 3$ \\
\hline B22 & 376.2477 & $\mathrm{C} 17 \mathrm{H} 18 \mathrm{BrN} 3 \mathrm{O} 2$ & 257.082 & $\mathrm{C} 10 \mathrm{H} 9 \mathrm{BrO} 3$ \\
\hline B23 & 385.4601 & C20H27N5O3 & 266.297 & C13H18N2O4 \\
\hline B24 & 335.3996 & $\mathrm{C} 2 \mathrm{OH} 21 \mathrm{~N} 3 \mathrm{O} 2$ & 216.24 & C13H12O3 \\
\hline C1 & 316.3119 & C15H16N4O4 & 197.1487 & C8H7NO5 \\
\hline
\end{tabular}




\begin{tabular}{|c|c|c|c|c|}
\hline $\mathrm{C} 2$ & 312.3663 & C17H20N4O2 & 193.201 & $\mathrm{C} 10 \mathrm{H} 11 \mathrm{NO} 3$ \\
\hline C3 & 301.4066 & C16H19N3OS & 182.243 & C9H10O2S \\
\hline C4 & 270.3296 & $\mathrm{C} 15 \mathrm{H} 18 \mathrm{~N} 4 \mathrm{O}$ & 151.164 & C8H9NO2 \\
\hline $\mathrm{C} 5$ & 382.4992 & $\mathrm{C} 22 \mathrm{H} 30 \mathrm{~N} 4 \mathrm{O} 2$ & 263.335 & $\mathrm{C} 15 \mathrm{H} 21 \mathrm{NO} 3$ \\
\hline C6 & 397.8581 & C20H20ClN5O2 & 278.694 & C13H11ClN2O3 \\
\hline C7 & 418.4882 & $\mathrm{C} 24 \mathrm{H} 26 \mathrm{~N} 4 \mathrm{O} 3$ & 299.324 & C17H17NO4 \\
\hline
\end{tabular}




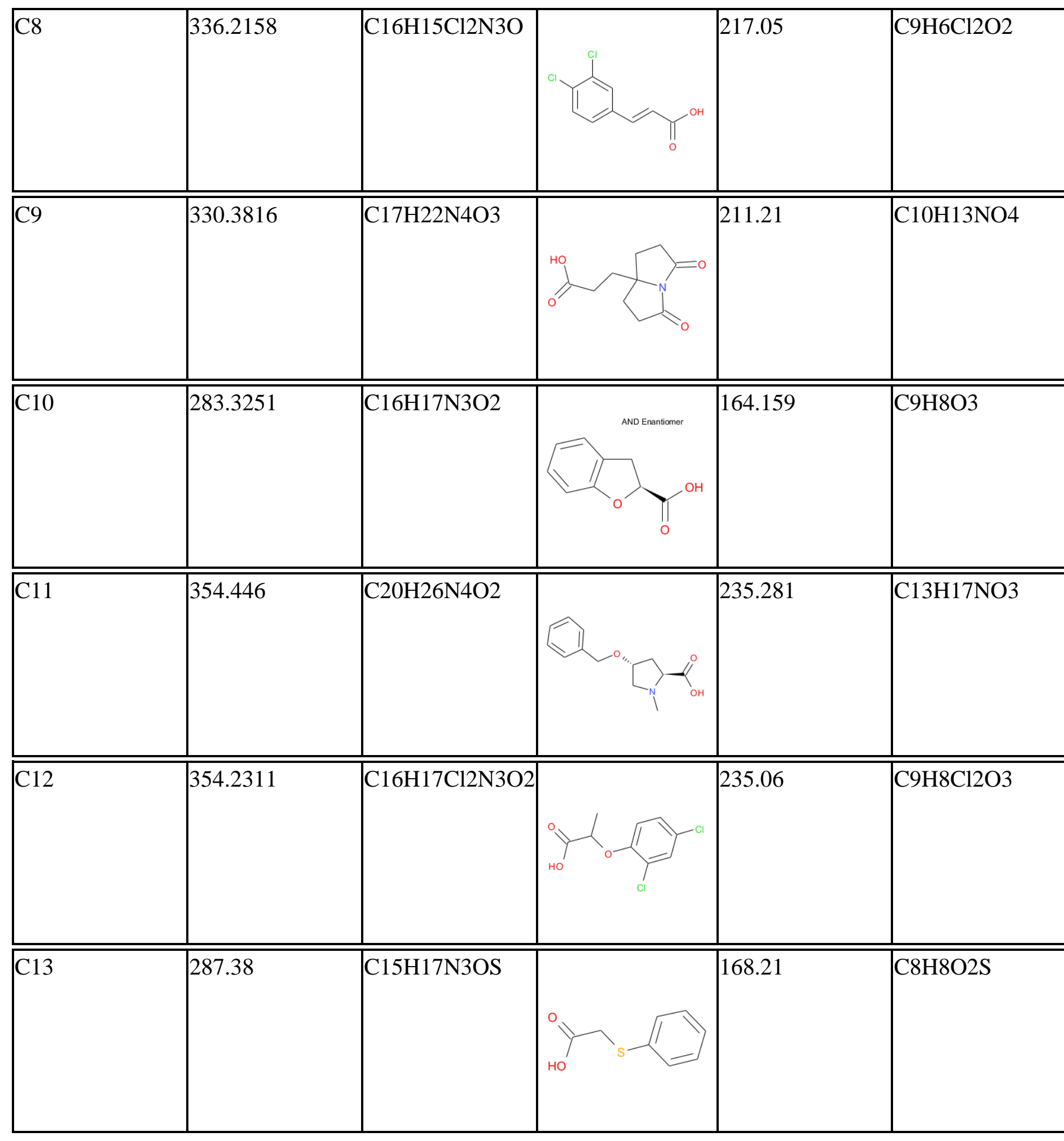




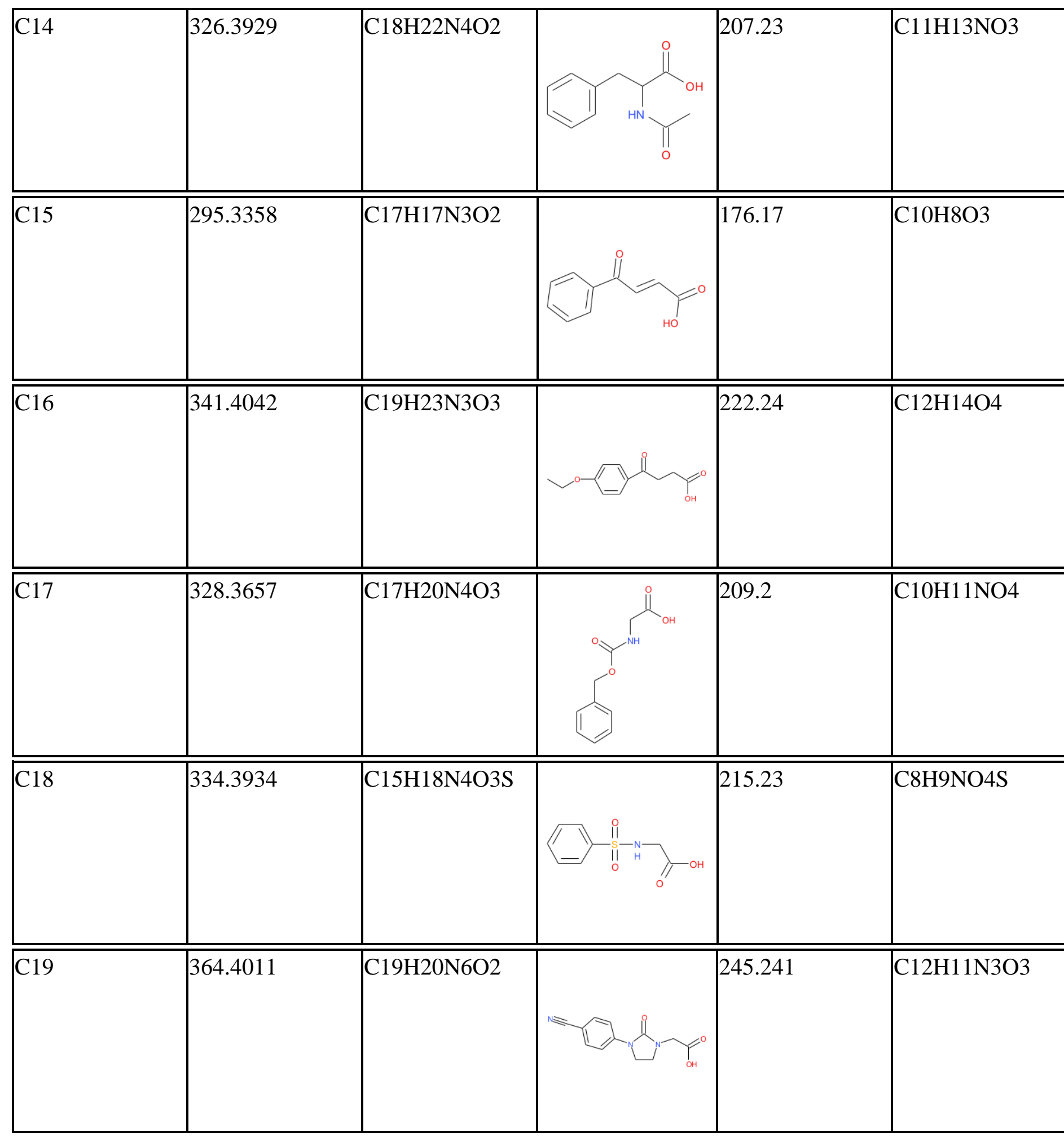




\begin{tabular}{|c|c|c|c|c|}
\hline C20 & 347.4319 & $\mathrm{C} 17 \mathrm{H} 21 \mathrm{~N} 3 \mathrm{O} 3 \mathrm{~S}$ & 228.267 & $\mathrm{C} 10 \mathrm{H} 12 \mathrm{O} 4 \mathrm{~S}$ \\
\hline C21 & 336.4292 & $\mathrm{C} 17 \mathrm{H} 28 \mathrm{~N} 4 \mathrm{O} 3$ & 217.3 & C10H19NO4 \\
\hline $\mathrm{C} 22$ & 397.8581 & $\mathrm{C} 20 \mathrm{H} 20 \mathrm{ClN} 5 \mathrm{O} 2$ & 278.694 & C13H11CIN2O3 \\
\hline C23 & 273.3054 & C15H16FN3O & 154.139 & C8H7FO2 \\
\hline C24 & 327.4207 & $\mathrm{C} 19 \mathrm{H} 25 \mathrm{~N} 3 \mathrm{O} 2$ & 208.257 & $\mathrm{C} 12 \mathrm{H} 16 \mathrm{O} 3$ \\
\hline D1 & 314.3391 & $\mathrm{C} 16 \mathrm{H} 18 \mathrm{~N} 4 \mathrm{O} 3$ & 195.173 & C9H9NO4 \\
\hline
\end{tabular}




\begin{tabular}{|c|c|c|c|c|}
\hline D2 & 402.4625 & $\mathrm{C} 21 \mathrm{H} 27 \mathrm{FN} 4 \mathrm{O} 3$ & 283.303 & C14H18FNO4 \\
\hline D3 & 373.4476 & $\mathrm{C} 23 \mathrm{H} 23 \mathrm{~N} 3 \mathrm{O} 2$ & 254.28 & C16H14O3 \\
\hline D4 & 314.3391 & C16H18N4O3 & 195.173 & C9H9NO4 \\
\hline D5 & 299.3245 & C16H17N3O3 & 180.158 & С9H8O4 \\
\hline D6 & 285.341 & $\mathrm{C} 16 \mathrm{H} 19 \mathrm{~N} 3 \mathrm{O} 2$ & 166.175 & С9H10O3 \\
\hline D7 & 348.4399 & C18H28N4O3 & 229.274 & C11H19NO4 \\
\hline
\end{tabular}




\begin{tabular}{|c|c|c|c|c|}
\hline D8 & 356.4189 & $\mathrm{C} 19 \mathrm{H} 24 \mathrm{~N} 4 \mathrm{O} 3$ & 237.255 & $\mathrm{C} 12 \mathrm{H} 15 \mathrm{NO} 4$ \\
\hline D9 & 308.3776 & $\mathrm{C} 18 \mathrm{H} 20 \mathrm{~N} 4 \mathrm{O}$ & 189.213 & C11H11NO2 \\
\hline D10 & 347.4103 & $\mathrm{C} 21 \mathrm{H} 21 \mathrm{~N} 3 \mathrm{O} 2$ & 228.246 & C14H12O3 \\
\hline D11 & 391.4878 & $\mathrm{C} 18 \mathrm{H} 25 \mathrm{~N} 5 \mathrm{O} 3 \mathrm{~S}$ & 272.323 & $\mathrm{C} 11 \mathrm{H} 16 \mathrm{~N} 2 \mathrm{O} 4 \mathrm{~S}$ \\
\hline D12 & 345.3929 & C18H23N3O4 & 226.227 & C11H14O5 \\
\hline D13 & 292.3767 & $\mathrm{C} 15 \mathrm{H} 24 \mathrm{~N} 4 \mathrm{O} 2$ & 173.212 & C8H15NO3 \\
\hline
\end{tabular}




\begin{tabular}{|c|c|c|c|c|}
\hline D14 & 363.428 & $\mathrm{C} 22 \mathrm{H} 22 \mathrm{FN} 3 \mathrm{O}$ & 244.27 & C15H13FO2 \\
\hline D15 & 331.4109 & $\mathrm{C} 21 \mathrm{H} 21 \mathrm{~N} 3 \mathrm{O}$ & 212.247 & $\mathrm{C} 14 \mathrm{H} 12 \mathrm{O} 2$ \\
\hline D16 & 295.3788 & $\mathrm{C} 18 \mathrm{H} 21 \mathrm{~N} 3 \mathrm{O}$ & 176.214 & C11H12O2 \\
\hline D17 & 306.3602 & C15H22N4O3 & 187.2 & C8H13NO4 \\
\hline D18 & 285.341 & $\mathrm{C} 16 \mathrm{H} 19 \mathrm{~N} 3 \mathrm{O} 2$ & 166.175 & С9H10O3 \\
\hline D19 & 326.416 & C17H18N4OS & 207.252 & $\mathrm{C} 10 \mathrm{H} 9 \mathrm{NO} 2 \mathrm{~S}$ \\
\hline
\end{tabular}




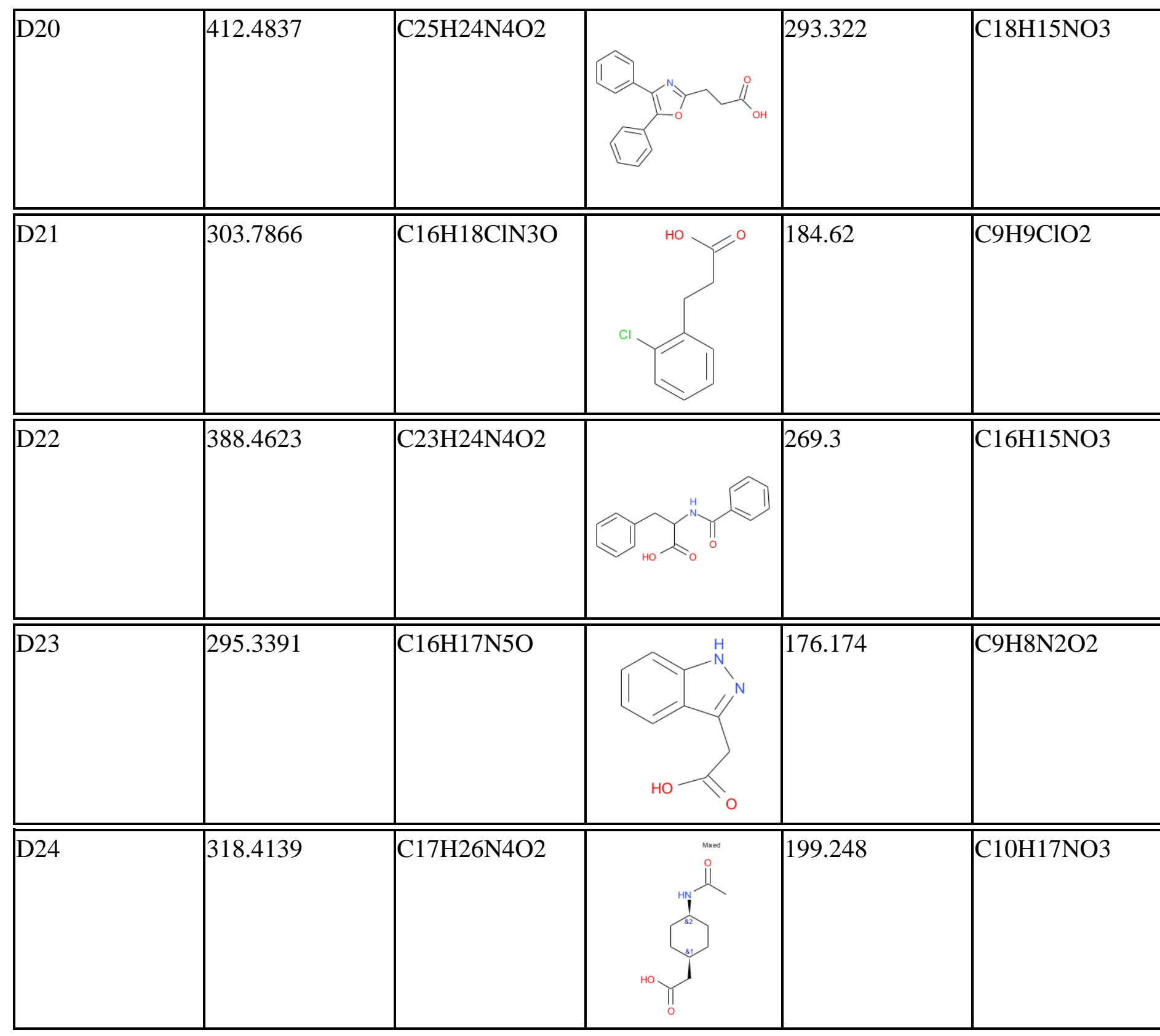




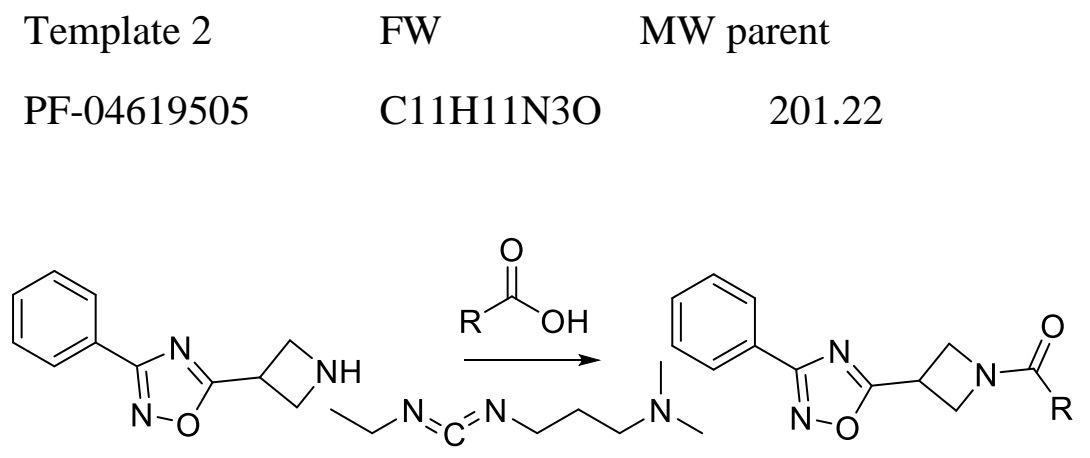

\begin{tabular}{|c|c|c|c|c|c|}
\hline Location & Product MW & Product FW & Monomer & Monomer MW & Monomer FW \\
\hline E1 & 441.4785 & $\mathrm{C} 26 \mathrm{H} 23 \mathrm{~N} 3 \mathrm{O} 4$ & & 258.28 & C15H14O4 \\
\hline E2 & 395.4531 & $\mathrm{C} 25 \mathrm{H} 21 \mathrm{~N} 3 \mathrm{O} 2$ & & 212.247 & C14H12O2 \\
\hline E3 & 398.4555 & $\mathrm{C} 21 \mathrm{H} 26 \mathrm{~N} 4 \mathrm{O} 4$ & & 215.247 & $\mathrm{C} 10 \mathrm{H} 17 \mathrm{NO} 4$ \\
\hline
\end{tabular}




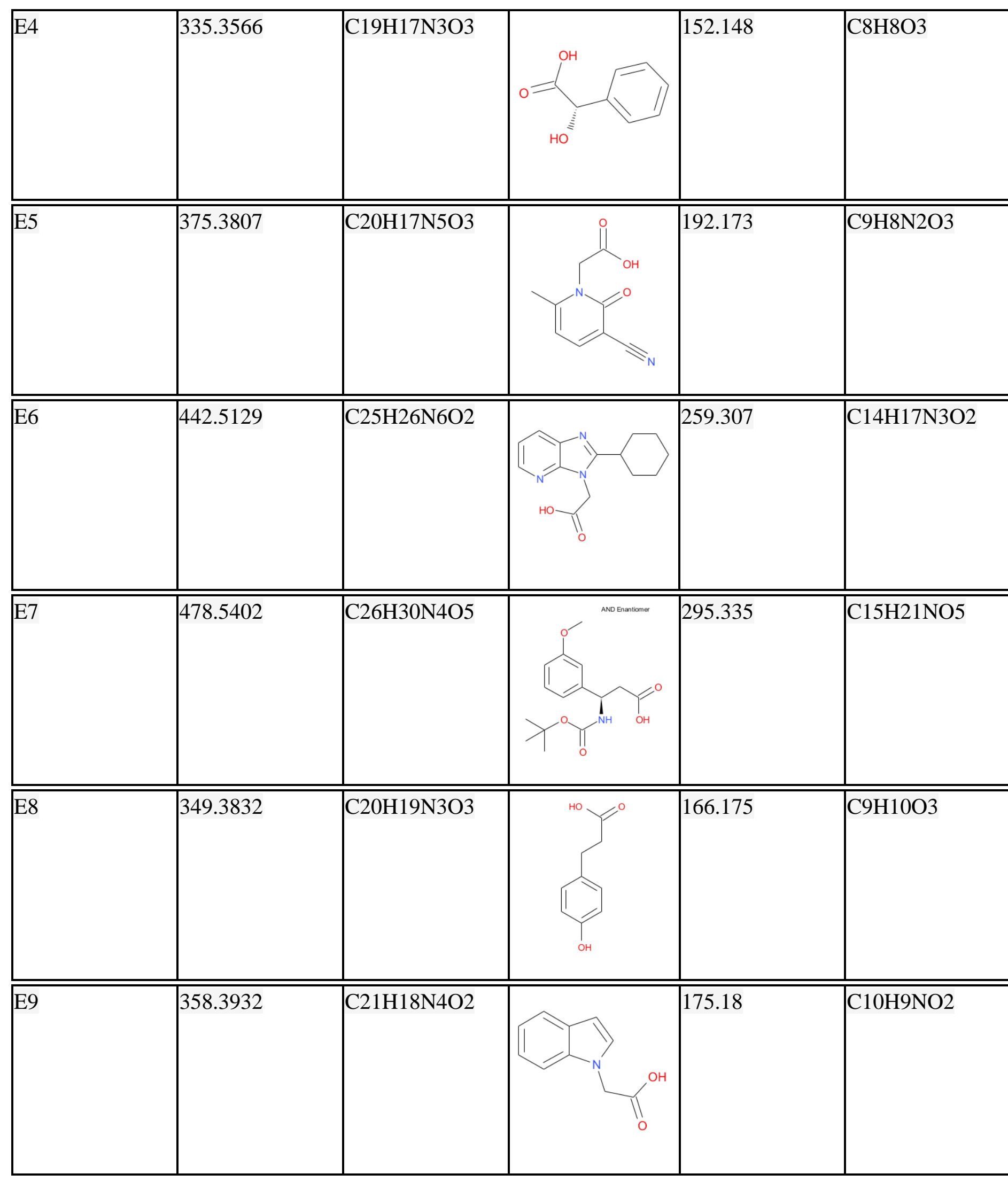




\begin{tabular}{|c|c|c|c|c|}
\hline E10 & 452.5261 & $\mathrm{C} 23 \mathrm{H} 24 \mathrm{~N} 4 \mathrm{O} 4 \mathrm{~S}$ & 269.32 & $\mathrm{C} 12 \mathrm{H} 15 \mathrm{NO} 4 \mathrm{~S}$ \\
\hline E11 & 446.4983 & $\mathrm{C} 25 \mathrm{H} 26 \mathrm{~N} 4 \mathrm{O} 4$ & 263.3 & C14H17NO4 \\
\hline E12 & 353.8022 & C19H16ClN3O2 & 170.594 & $\mathrm{C} 8 \mathrm{H} 7 \mathrm{ClO} 2$ \\
\hline E13 & 469.5317 & C28H27N3O4 & 286.35 & C17H18O4 \\
\hline E14 & 395.8389 & $\mathrm{C} 21 \mathrm{H} 18 \mathrm{ClN} 3 \mathrm{O} 3$ & 212.63 & $\mathrm{C} 10 \mathrm{H} 9 \mathrm{ClO} 3$ \\
\hline E15 & 441.4785 & C26H23N3O4 & 258.272 & C15H14O4 \\
\hline
\end{tabular}




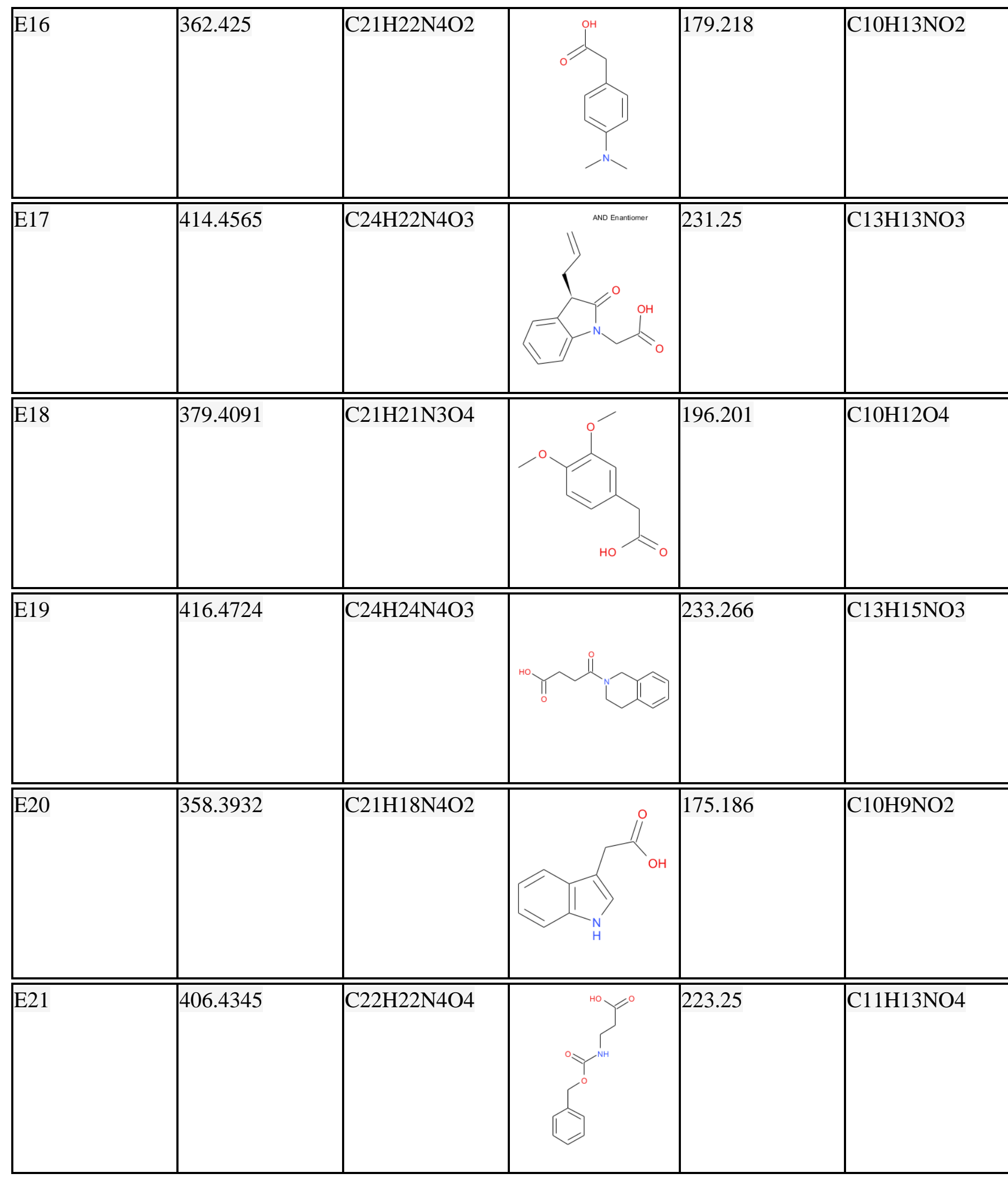




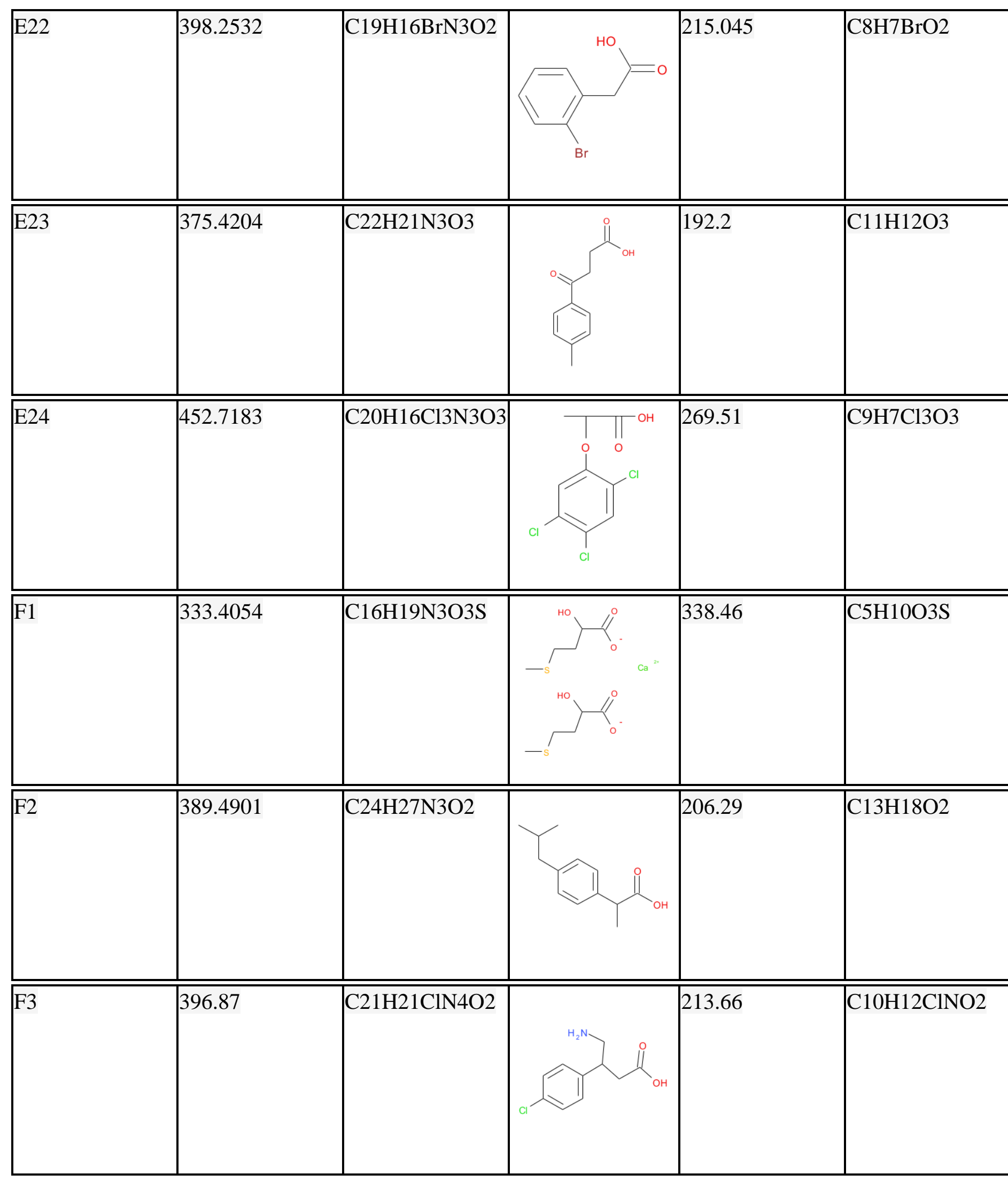




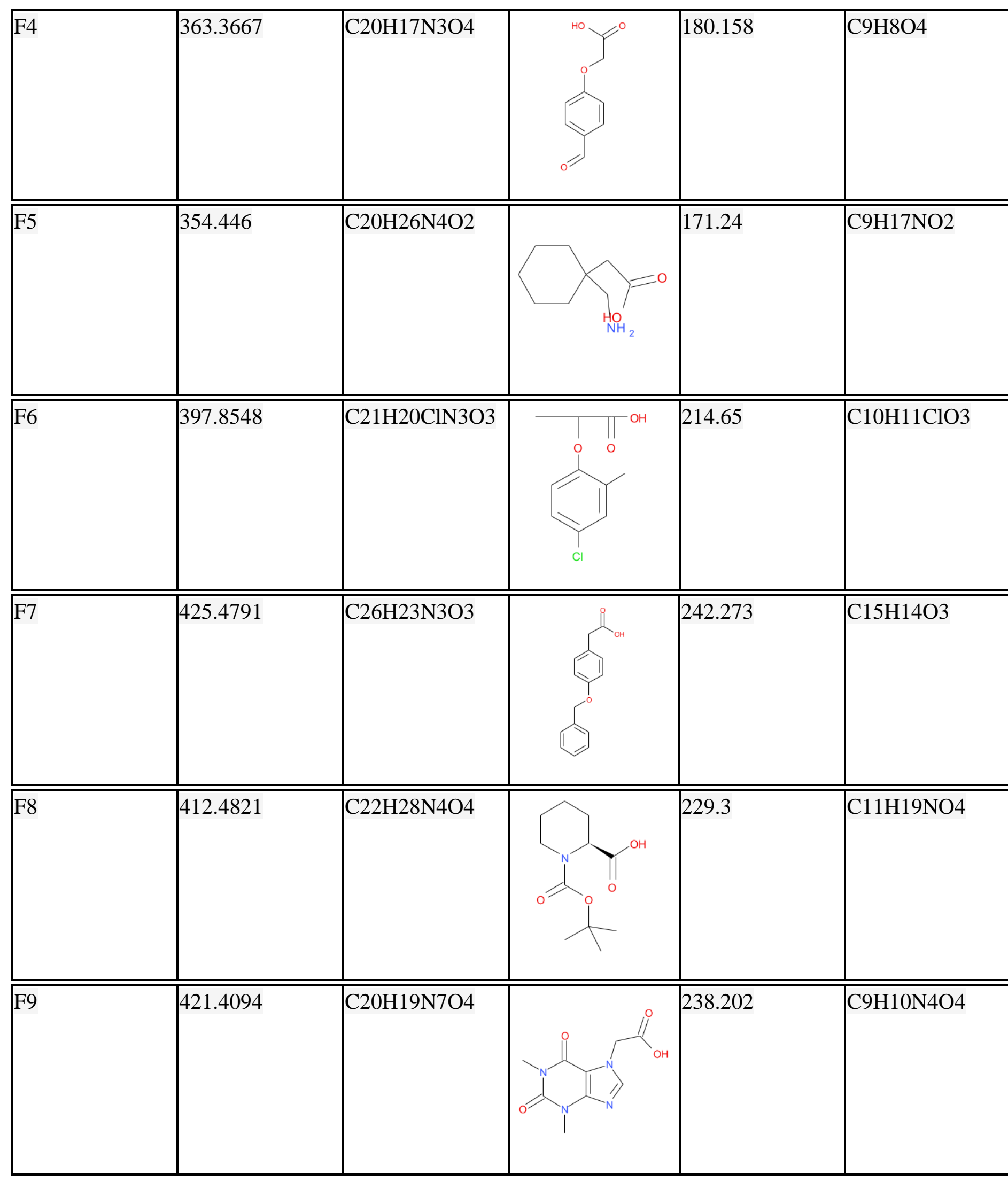




\begin{tabular}{|c|c|c|c|c|}
\hline F10 & 459.9242 & $\mathrm{C} 26 \mathrm{H} 22 \mathrm{ClN} 3 \mathrm{O} 3$ & 276.718 & $\mathrm{C} 15 \mathrm{H} 13 \mathrm{ClO} 3$ \\
\hline F11 & 375.4204 & $\mathrm{C} 22 \mathrm{H} 21 \mathrm{~N} 3 \mathrm{O} 3$ & |192.214 & C11H12O3 \\
\hline F12 & 362.3819 & $\mathrm{C} 20 \mathrm{H} 18 \mathrm{~N} 4 \mathrm{O} 3$ & 179.174 & C9H9NO3 \\
\hline F13 & 333.3838 & $\mathrm{C} 20 \mathrm{H} 19 \mathrm{~N} 3 \mathrm{O} 2$ & 150.176 & C9H10O2 \\
\hline F14 & 354.3599 & C18H18N4O4 & 171.151 & C7H9NO4 \\
\hline F15 & 430.3799 & C21H17F3N4O3 & 247.172 & $\mathrm{C} 10 \mathrm{H} 8 \mathrm{~F} 3 \mathrm{NO} 3$ \\
\hline
\end{tabular}




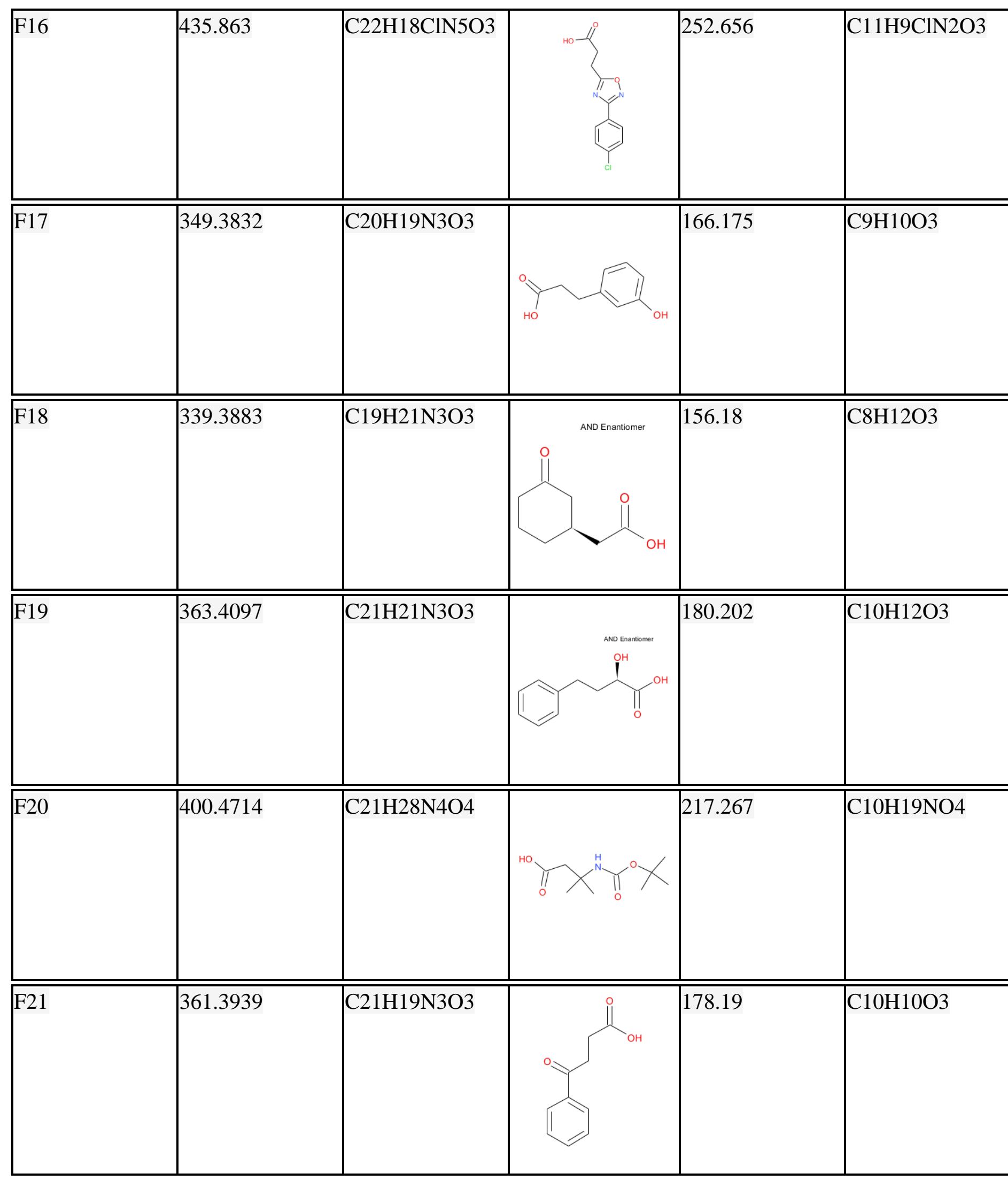




\begin{tabular}{|c|c|c|c|c|}
\hline F22 & 440.2899 & $\mathrm{C} 21 \mathrm{H} 18 \mathrm{BrN} 3 \mathrm{O} 3$ & 257.082 & $\mathrm{C} 10 \mathrm{H} 9 \mathrm{BrO} 3$ \\
\hline F23 & 449.5023 & C24H27N5O4 & 266.297 & C13H18N2O4 \\
\hline F24 & 399.4418 & $\mathrm{C} 24 \mathrm{H} 21 \mathrm{~N} 3 \mathrm{O} 3$ & 216.24 & C13H12O3 \\
\hline G1 & 380.3541 & C19H16N4O5 & 197.1487 & C8H7NO5 \\
\hline G2 & 376.4085 & $\mathrm{C} 21 \mathrm{H} 20 \mathrm{~N} 4 \mathrm{O} 3$ & 193.201 & $\mathrm{C} 10 \mathrm{H} 11 \mathrm{NO} 3$ \\
\hline G3 & 365.4488 & C20H19N3O2S & 182.243 & C9H10O2S \\
\hline
\end{tabular}




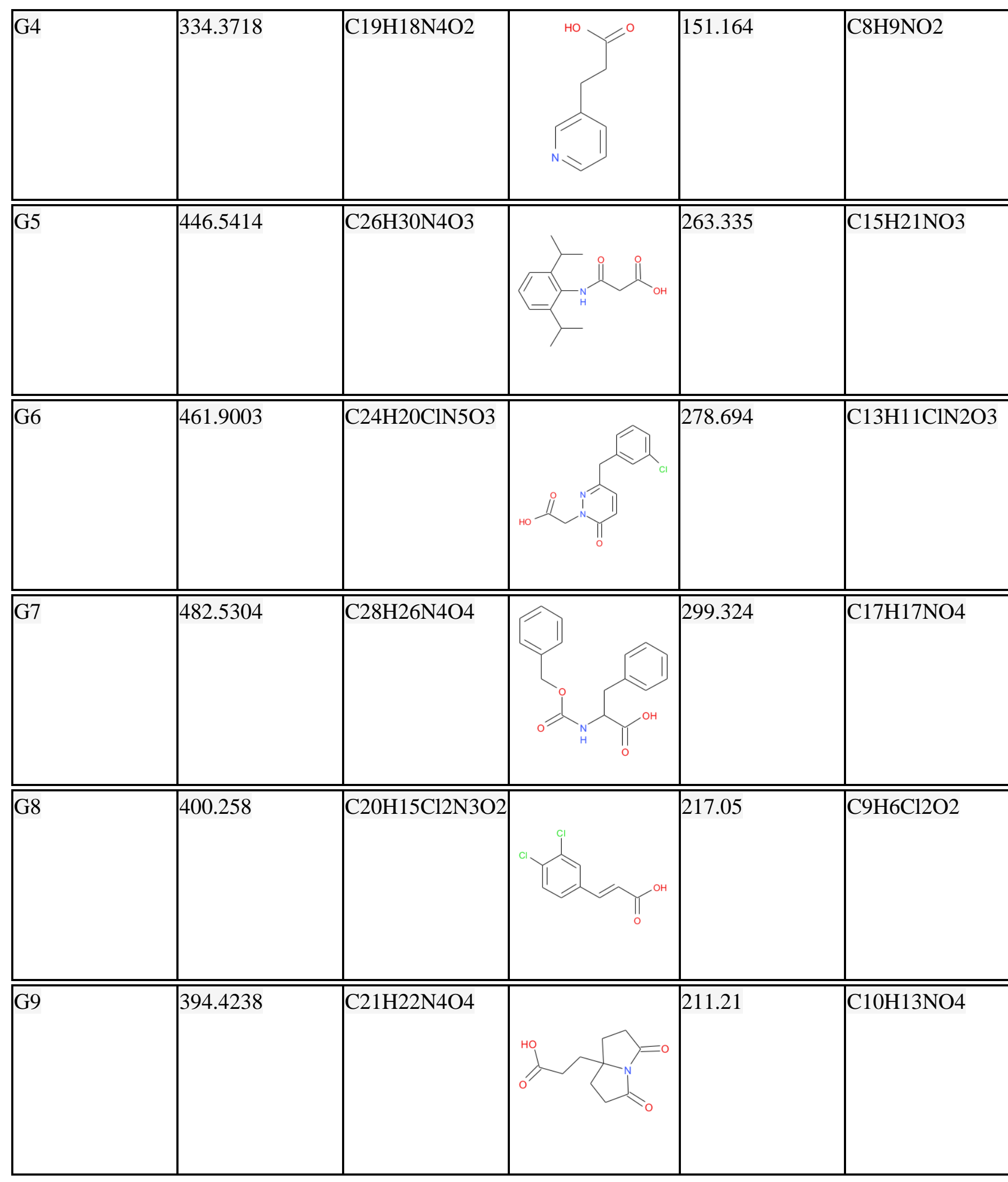




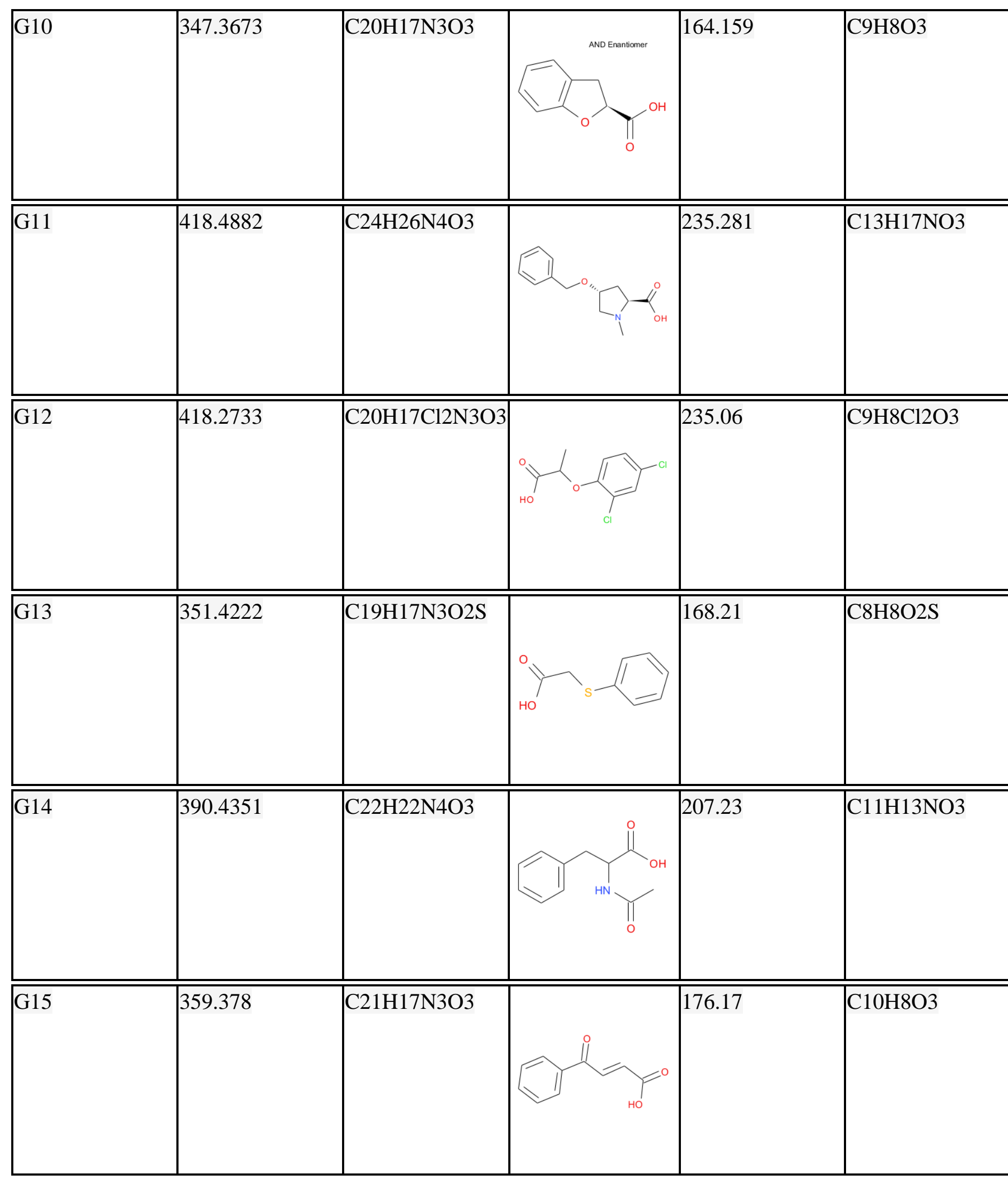




\begin{tabular}{|c|c|c|c|c|}
\hline G16 & 405.4464 & C23H23N3O4 & 222.24 & C12H14O4 \\
\hline G17 & 392.4079 & C21H20N4O4 & 209.2 & C10H11NO4 \\
\hline G18 & 398.4356 & C19H18N4O4S & 215.23 & C8H9NO4S \\
\hline G19 & 428.4433 & C23H20N6O3 & 245.241 & $\mathrm{C} 12 \mathrm{H} 11 \mathrm{~N} 3 \mathrm{O} 3$ \\
\hline G20 & 411.4741 & $\mathrm{C} 21 \mathrm{H} 21 \mathrm{~N} 3 \mathrm{O} 4 \mathrm{~S}$ & 228.267 & $\mathrm{C} 10 \mathrm{H} 12 \mathrm{O} 4 \mathrm{~S}$ \\
\hline G21 & 400.4714 & C21H28N4O4 & 217.3 & C10H19NO4 \\
\hline
\end{tabular}




\begin{tabular}{|c|c|c|c|c|}
\hline $\mathrm{G} 22$ & 461.9003 & $\mathrm{C} 24 \mathrm{H} 20 \mathrm{ClN} 5 \mathrm{O} 3$ & 278.694 & $\mathrm{C} 13 \mathrm{H} 11 \mathrm{ClN} 2 \mathrm{O} 3$ \\
\hline G23 & 337.3476 & C19H16FN3O2 & 154.139 & C8H7FO2 \\
\hline G24 & 391.4629 & $\mathrm{C} 23 \mathrm{H} 25 \mathrm{~N} 3 \mathrm{O} 3$ & 208.257 & C12H16O3 \\
\hline H1 & 378.3813 & C20H18N4O4 & 195.173 & C9H9NO4 \\
\hline $\mathrm{H} 2$ & 466.5047 & C25H27FN4O4 & 283.303 & C14H18FNO4 \\
\hline H3 & 437.4898 & $\mathrm{C} 27 \mathrm{H} 23 \mathrm{~N} 3 \mathrm{O} 3$ & 254.28 & C16H14O3 \\
\hline
\end{tabular}




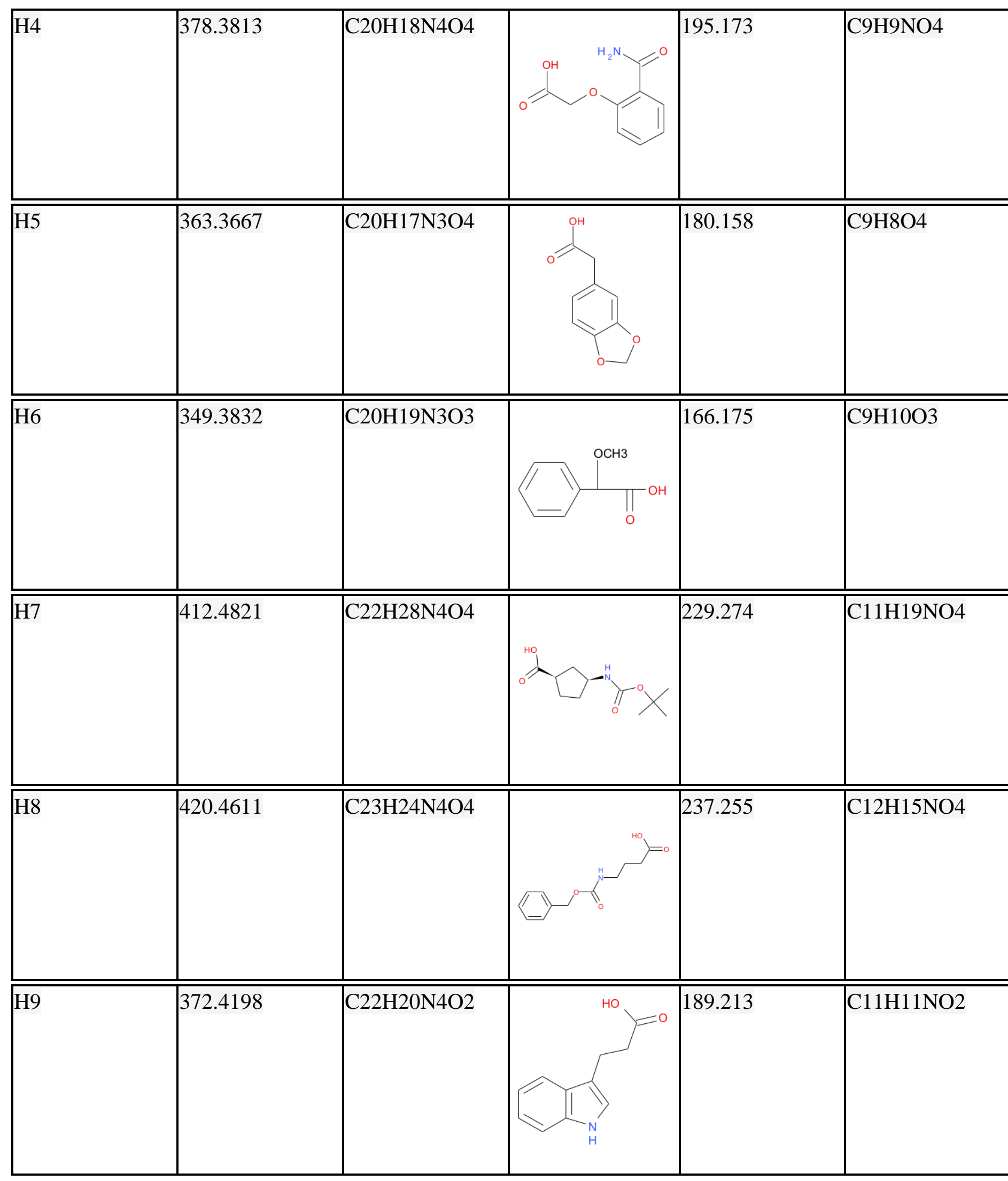




\begin{tabular}{|c|c|c|c|c|}
\hline H10 & 411.4525 & $\mathrm{C} 25 \mathrm{H} 21 \mathrm{~N} 3 \mathrm{O} 3$ & 228.246 & C14H12O3 \\
\hline H11 & 455.53 & $\mathrm{C} 22 \mathrm{H} 25 \mathrm{~N} 5 \mathrm{O} 4 \mathrm{~S}$ & 272.323 & C11H16N2O4S \\
\hline H12 & 409.4351 & $\mathrm{C} 22 \mathrm{H} 23 \mathrm{~N} 3 \mathrm{O} 5$ & 226.227 & C11H14O5 \\
\hline H13 & 356.4189 & C19H24N4O3 & 173.212 & C8H15NO3 \\
\hline H14 & 427.4702 & $\mathrm{C} 26 \mathrm{H} 22 \mathrm{FN} 3 \mathrm{O} 2$ & 244.27 & C15H13FO2 \\
\hline H15 & 395.4531 & $\mathrm{C} 25 \mathrm{H} 21 \mathrm{~N} 3 \mathrm{O} 2$ & 212.247 & C14H12O2 \\
\hline
\end{tabular}




\begin{tabular}{|c|c|c|c|c|}
\hline H16 & 359.421 & $\mathrm{C} 22 \mathrm{H} 21 \mathrm{~N} 3 \mathrm{O} 2$ & 176.214 & C11H12O2 \\
\hline H17 & 370.4024 & C19H22N4O4 & 187.2 & C8H13NO4 \\
\hline H18 & 349.3832 & C20H19N3O3 & 166.175 & C9H10O3 \\
\hline H19 & 390.4582 & $\mathrm{C} 21 \mathrm{H} 18 \mathrm{~N} 4 \mathrm{O} 2 \mathrm{~S}$ & 207.252 & C10H9NO2S \\
\hline $\mathrm{H} 20$ & 476.5259 & $\mathrm{C} 29 \mathrm{H} 24 \mathrm{~N} 4 \mathrm{O} 3$ & 293.322 & C18H15NO3 \\
\hline H21 & 367.8288 & $\mathrm{C} 20 \mathrm{H} 18 \mathrm{ClN} 3 \mathrm{O} 2$ & 184.62 & C9H9ClO2 \\
\hline
\end{tabular}




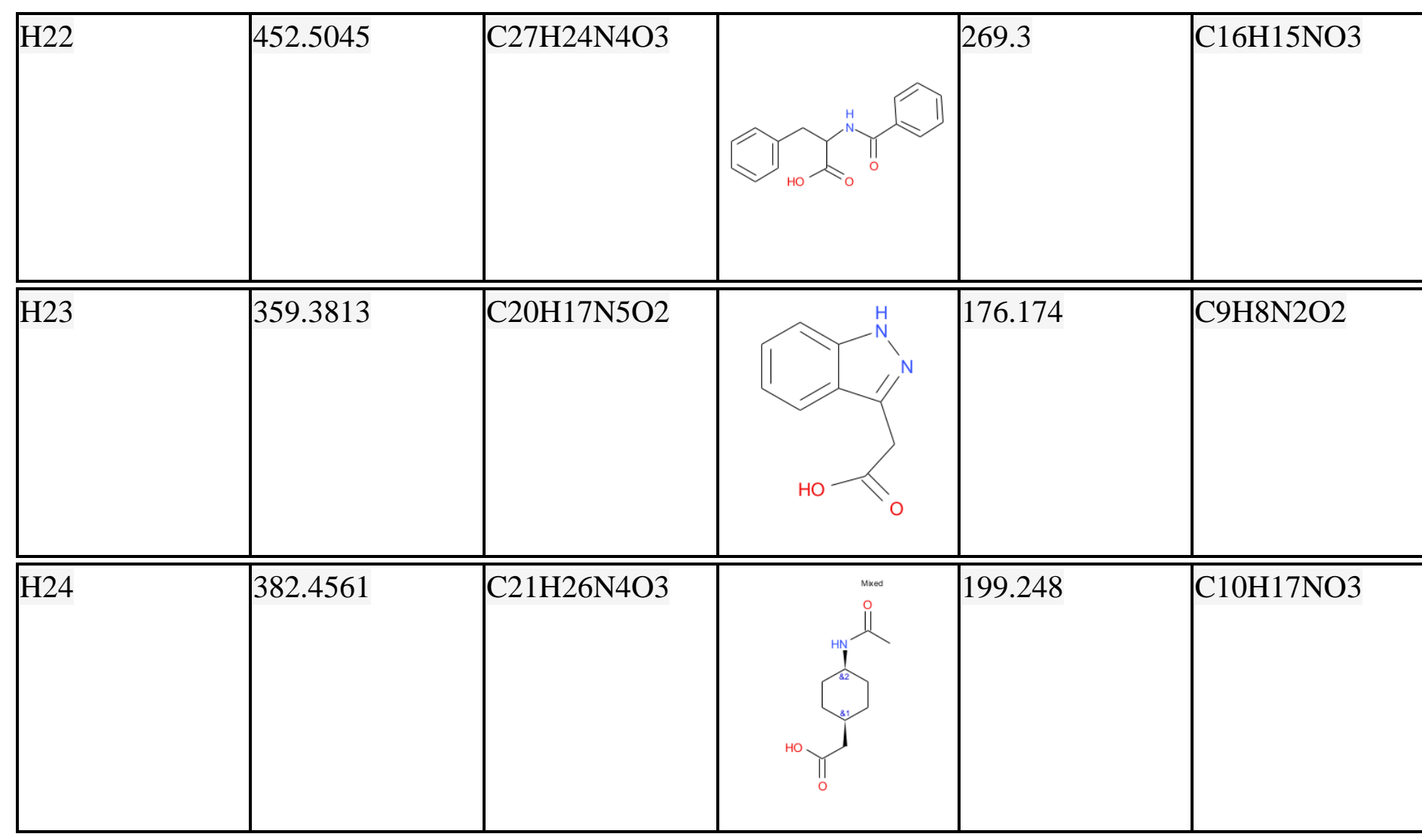


Supplemental Table 4. Raw UPLC-MS data used for visual plots

Experiment 2: Pd-catalyzed C-N formation PMC screen

\begin{tabular}{|l|l|l|}
\hline Location & $\begin{array}{l}\text { TIC AreaAbs } \\
\text { UPLC-MS }\end{array}$ & $\begin{array}{l}\text { Peak area ADE- } \\
\text { OPI-MS }\end{array}$ \\
\hline A1 & 4041808.794 & 1082636.864 \\
\hline A2 & 3124121.67 & 2172470.347 \\
\hline A3 & 5141439 & 295474.6136 \\
\hline A4 & 935162.7 & 295309.6551 \\
\hline A5 & 611805 & 798513.6265 \\
\hline A6 & 4600072.7 & 2292050.04 \\
\hline A7 & 2697008.7 & 1112733.908 \\
\hline A8 & 605040.1 & 817482.5875 \\
\hline A9 & 1463688.9 & 1589712.409 \\
\hline A10 & 2121765.4 & 1826085.096 \\
\hline A11 & 2298418.9 & 1574636.732 \\
\hline A12 & 1396779.3 & 1198594.021 \\
\hline A13 & 1175970 & 521918.555 \\
\hline A14 & 1190688 & 701973.6102 \\
\hline A15 & 1464462.8 & 1045898.959 \\
\hline A16 & 2218297.6 & 1229617.173 \\
\hline A17 & 1450327.8 & 1069925.319 \\
\hline A18 & 1193591.8 & 1379819.854 \\
\hline A19 & 1357214.5 & 1025303.582 \\
\hline A20 & 0 & 22206.79905 \\
\hline A21 & 367493.77 & 955236.1845 \\
\hline A22 & 604450.2 & 807014.3024 \\
\hline A23 & 1154668.65 & 1146653.46 \\
\hline A24 & 0 & 13.674 \\
\hline B1 & 0 & 56.4271848 \\
\hline B2 & 3011993.6 & 2107069.003 \\
\hline B3 & 0 & 15.01504514 \\
\hline B4 & 8820045 & 870116.1132 \\
\hline B5 & 0 & 75.7289423 \\
\hline B6 & 1115363.1 & 1121005.052 \\
\hline B7 & 0 & 12509.18699 \\
\hline B8 & 1089092.1 & 398661.866 \\
\hline B9 & 0 & 17.12778738 \\
\hline B10 & 1712493.3 & 1210934.505 \\
\hline B11 & 2193957.7 & 1574506.43 \\
\hline B12 & 1240255.3 & 657341.7171 \\
\hline B13 & 1691961.3 & 1871315.928 \\
\hline B14 & 562575.8 & 1017048.002 \\
\hline & & \\
\hline
\end{tabular}




\begin{tabular}{|l|l|l|}
\hline B15 & 1712845.2 & 989903.9495 \\
\hline B16 & 1213988.5 & 939656.4634 \\
\hline B17 & 1190355.1 & 803527.0865 \\
\hline B18 & 1099258.9 & 1138088.638 \\
\hline B19 & 1569655 & 827553.4702 \\
\hline B20 & 0 & 14762.44949 \\
\hline B21 & 2467177.7 & 1335704.269 \\
\hline B22 & 7030653.2 & 548521.7213 \\
\hline B23 & 7122578.9 & 2027020.042 \\
\hline B24 & 1545809.1 & 961748.138 \\
\hline C1 & 1388316.4 & 1250750.203 \\
\hline C2 & 1322091.9 & 1204618.177 \\
\hline C3 & 1744591.8 & 1305766.353 \\
\hline C4 & 0 & 1556282.01 \\
\hline C5 & 2549634.5 & 2152205.089 \\
\hline C6 & 1295717.7 & 775099.125 \\
\hline C7 & 3633119.6 & 1604993.256 \\
\hline C8 & 1013389.4 & 632968.7451 \\
\hline C9 & 627257.7 & 1202903.705 \\
\hline C10 & 1515597.2 & 1257408.948 \\
\hline C11 & 6884477.3 & 3327709.484 \\
\hline C12 & 1645853.9 & 1075055.837 \\
\hline C13 & 1372262.3 & 1237977.694 \\
\hline C14 & 1062343.2 & 940200.0707 \\
\hline C15 & 885030.9 & 555162.2948 \\
\hline C16 & 0 & 28.8267988 \\
\hline C17 & 1408089 & 879821.0136 \\
\hline C18 & 1166097 & 843169.5738 \\
\hline C19 & 1215568.7 & 1100891.601 \\
\hline C20 & 0 & 22418.19496 \\
\hline C21 & 1663330 & 684911.815 \\
\hline C22 & 1565022.8 & 993977.8656 \\
\hline C23 & 2009571.8 & 1516561.271 \\
\hline C24 & 1920144.6 & 879234.4766 \\
\hline D1 & 2129846.9 & 1608796.809 \\
\hline D2 & 1471655.3 & 532682.5195 \\
\hline D3 & 1549853.5 & 1051370.755 \\
\hline D4 & 9070008.9 & 842843.6549 \\
\hline D5 & 1154254.6 & 1287159.09 \\
\hline D6 & 1530840.8 & 999676.927 \\
\hline D7 & 1743828 & 799904.4442 \\
\hline D8 & 1446919.9 & 1170259.946 \\
\hline D9 & 1480846.9 & 1232841.854 \\
\hline & & \\
\hline
\end{tabular}




\begin{tabular}{|l|l|l|}
\hline D10 & 2877991.8 & 1589132.885 \\
\hline D11 & 4401361.9 & 1297286.531 \\
\hline D12 & 1544025.4 & 1387241.089 \\
\hline D13 & 899104.1 & 817705.264 \\
\hline D14 & 2004796.8 & 1269607.512 \\
\hline D15 & 1642678.9 & 1589739.369 \\
\hline D16 & 2033987.1 & 1205076.428 \\
\hline D17 & 908859.8 & 888080.2148 \\
\hline D18 & 1510141.6 & 1626886.095 \\
\hline D19 & 1687640.9 & 1253717.817 \\
\hline D20 & 265694.9 & 51492.76423 \\
\hline D21 & 1339536 & 970347.2691 \\
\hline D22 & 3023715.2 & 1179906.268 \\
\hline D23 & 1185187.9 & 579033.7145 \\
\hline D24 & 0 & 49.17866421 \\
\hline E1 & 0 & 313717.2571 \\
\hline E2 & 0 & 1319147.27 \\
\hline E3 & 0 & 75580.1843 \\
\hline E4 & 530368 & 184935.5749 \\
\hline E5 & 429541 & 234395.5119 \\
\hline E6 & 6329162 & 69.88777011 \\
\hline E7 & 0 & 329631.6503 \\
\hline E8 & 587901 & 296719.5333 \\
\hline E9 & 498291 & 823663.8095 \\
\hline E10 & 1365179.5 & 885344.5968 \\
\hline E11 & 891120 & 762779.3609 \\
\hline E12 & 416499.2 & 534332.7133 \\
\hline E13 & 523835 & 139969.1839 \\
\hline E14 & 618845 & 245046.2502 \\
\hline E15 & 717174.4 & 405487.1722 \\
\hline E16 & 2442611 & 606681.0381 \\
\hline E17 & 1164983.8 & 494647.6946 \\
\hline E18 & 642528.8 & 556167.7003 \\
\hline E19 & 843034.2 & 320547.7575 \\
\hline E20 & 0 & 10930.40058 \\
\hline E21 & 613560.4 & 321565.351 \\
\hline E22 & 0 & 385788.4963 \\
\hline E23 & 948175.4 & 466391.1915 \\
\hline E24 & 0 & 110904.4435 \\
\hline F1 & 0 & 10276.68191 \\
\hline F2 & 1421834 & 781990.675 \\
\hline F3 & 0 & 0 \\
\hline F4 & 312233 & 203210.6685 \\
\hline & & \\
\hline
\end{tabular}




\begin{tabular}{|l|l|l|}
\hline F5 & 0 & 396.4982442 \\
\hline F6 & 745985.9 & 352353.8513 \\
\hline F7 & 0 & 2385.161488 \\
\hline F8 & 0 & 89476.95481 \\
\hline F9 & 283950 & 45778.73566 \\
\hline F10 & 0 & 423799.6336 \\
\hline F11 & 746516.8 & 569253.8741 \\
\hline F12 & 778753.7 & 285763.9696 \\
\hline F13 & 678970.6 & 948149.0955 \\
\hline F14 & 609271.8 & 507973.1563 \\
\hline F15 & 806357.6 & 372569.0279 \\
\hline F16 & 0 & 354865.0549 \\
\hline F17 & 662828 & 307550.7914 \\
\hline F18 & 852579 & 790829.8801 \\
\hline F19 & 646818 & 296572.6202 \\
\hline F20 & 0 & 4359.430487 \\
\hline F21 & 982222.6 & 476580.2211 \\
\hline F22 & 315131.5 & 180168.8819 \\
\hline F23 & 6770759 & 1299830.017 \\
\hline F24 & 498765 & 434129.6419 \\
\hline G1 & 615734 & 474933.6048 \\
\hline G2 & 892880.9 & 425765.8051 \\
\hline G3 & 957649 & 658207.8608 \\
\hline G4 & 2774575 & 583503.4258 \\
\hline G5 & 1837888 & 903208.1014 \\
\hline G6 & 672811 & 360306.0706 \\
\hline G7 & 1210987 & 656056.4388 \\
\hline G8 & 0 & 121898.7049 \\
\hline G9 & 459606.6 & 378166.938 \\
\hline G10 & 389149.5 & 534010.8879 \\
\hline G11 & 6975449.2 & 1611294.907 \\
\hline G12 & 0 & 302137.488 \\
\hline G13 & 904247 & 613911.3775 \\
\hline G14 & 806979 & 349907.3834 \\
\hline G15 & 0 & 40868.2014 \\
\hline G16 & 0 & 9.288 \\
\hline G17 & 779163 & 375666.0377 \\
\hline G18 & 669454 & 452017.9758 \\
\hline G19 & 496446.8 & 345695.5528 \\
\hline G20 & 0 & 8373.300504 \\
\hline G21 & 0 & 194493.3132 \\
\hline G22 & 772055 & 527837.6571 \\
\hline G23 & 785450 & 708086.6086 \\
\hline & & \\
\hline
\end{tabular}




\begin{tabular}{|l|l|l|}
\hline G24 & 0 & 301858.4997 \\
\hline H1 & 523653 & 676114.2316 \\
\hline H2 & 0 & 147339.83 \\
\hline H3 & 1148281 & 340289.6299 \\
\hline H4 & 967848 & 428395.1948 \\
\hline H5 & 684107 & 610166.0263 \\
\hline H6 & 921437 & 759293.5017 \\
\hline H7 & 0 & 164983.6408 \\
\hline H8 & 763506 & 360258.0789 \\
\hline H9 & 722463 & 405850.0981 \\
\hline H10 & 0 & 674636.9528 \\
\hline H11 & 3005302 & 526835.6505 \\
\hline H12 & 767865 & 548190.4034 \\
\hline H13 & 763733 & 121262.5409 \\
\hline H14 & 962073 & 476718.319 \\
\hline H15 & 997737 & 579237.267 \\
\hline H16 & 563094 & 437913.5423 \\
\hline H17 & 614730 & 358977.8186 \\
\hline H18 & 834398 & 746775.7235 \\
\hline H19 & 1538269 & 723320.4635 \\
\hline H20 & 0 & 15764.22753 \\
\hline H21 & 474597 & 472619.4191 \\
\hline H22 & 0 & 20.124 \\
\hline H23 & 1543406 & 382591.4205 \\
\hline H24 & 0 & 630.6303351 \\
\hline & & \\
\hline
\end{tabular}


Supplemental Table 5. Plate mapping of acid reactant (monomer) and expected product molecular and formula weight

Experiment 3. DNA headpiece amidation reaction (wells A1-H12)

\begin{tabular}{|l|l|l|l|l|}
\hline Product_FW & Well & monomer_FW & Prod_MW & DNA_SM \\
\hline C169H227N52O104P17 & A1 & C15H12O3 & 5177.4843 & C154H215N52O101P17 \\
\hline C168H225N52O102P17 & A2 & C14H10O & 5131.4589 & \\
\hline C164H230N53O104P17 & A3 & C10H15NO3 & 5134.4613 & \\
\hline C162H221N52O103P17 & A4 & C8H6O2 & 5071.3623 & \\
\hline C163H221N54O103P17 & A5 & C9H6N2O2 & 5111.3864 & \\
\hline C168H230N55O102P17 & A6 & C14H15N3O & 5178.5187 & \\
\hline C169H234N53O105P17 & A7 & C15H19NO4 & 5214.5459 & \\
\hline C163H223N52O103P17 & A8 & C9H8O2 & 5085.3889 & \\
\hline C164H222N53O102P17 & A9 & C10H7NO & 5094.399 & \\
\hline C166H228N53O104P17S & A10 & C12H13NO3S & 5188.5318 & \\
\hline C168H230N53O104P17 & A11 & C14H15NO3 & 5182.5041 & \\
\hline C162H220C1N52O102P17 & A12 & C8H5C1O & 5089.808 & \\
\hline C171H231N52O104P17 & B1 & C17H16O3 & 5205.5374 & \\
\hline C164H222N52O103P17 & B2 & C10H7ClO2 & 5131.8447 & \\
\hline C169H227N52O104P17 & B3 & C15H12O3 & 5177.4843 & \\
\hline C164H226N53O102P17 & B4 & C10H11NO & 5098.4307 & \\
\hline C167H226N53O103P17 & B5 & C13H11NO2 & 5150.4622 & \\
\hline C164H225N52O104P17 & B6 & C10H10O3 & 5115.4149 & \\
\hline C167H228N53O103P17 & B7 & C13H13NO2 & 5152.4781 & \\
\hline
\end{tabular}




\begin{tabular}{|c|c|c|c|c|}
\hline Product_FW & Well & monomer_FW & Prod_MW & DNA_SM \\
\hline C164H222N53O102P17 & B8 & C10H7NO & 5094.399 & \\
\hline C165H226N53O104P17 & B9 & C11H11NO3 & 5142.4402 & \\
\hline C162H220BrN52O102P17 & B10 & $\mathrm{C} 8 \mathrm{H} 5 \mathrm{BrO}$ & 5134.259 & \\
\hline C165H225N52O103P17 & B11 & $\mathrm{C} 11 \mathrm{H} 10 \mathrm{O} 2$ & 5111.4262 & \\
\hline C163H220ClN52O103P17 & B12 & $\mathrm{C} 9 \mathrm{H} 5 \mathrm{Cl} 3 \mathrm{O} 2$ & 5188.7241 & \\
\hline C159H222N52O103SP17 & $\mathrm{C} 1$ & $\mathrm{C} 5 \mathrm{H} 7 \mathrm{O} 2 \mathrm{~S}$ & 5068.4032 & \\
\hline C167H231N52O102P17 & $\mathrm{C} 2$ & $\mathrm{C} 13 \mathrm{H} 16 \mathrm{O}$ & 5125.4958 & \\
\hline C164H225ClN53O102P17 & $\mathrm{C} 3$ & C10H10CINO & 5132.8758 & \\
\hline C163H221N52O104P17 & $\mathrm{C} 4$ & $\mathrm{C} 9 \mathrm{H} 6 \mathrm{O} 3$ & 5099.3724 & \\
\hline C163H230N53O102P17 & $\mathrm{C} 5$ & C9H15NO & 5090.4518 & \\
\hline C164H224CIN52O103P17 & C6 & $\mathrm{C} 10 \mathrm{H} 9 \mathrm{ClO} 2$ & 5133.8605 & \\
\hline C169H227N52O103P17 & C7 & $\mathrm{C} 15 \mathrm{H} 12 \mathrm{O} 2$ & 5161.4849 & \\
\hline C165H232N53O104P17 & $\mathrm{C} 8$ & C11H17NO3 & 5148.4879 & \\
\hline C163H223N56O104P17 & C9 & $\mathrm{C} 9 \mathrm{H} 8 \mathrm{~N} 4 \mathrm{O} 3$ & 5157.4151 & \\
\hline C169H226ClN52O103P17 & $\mathrm{C} 10$ & $\mathrm{C} 15 \mathrm{H} 11 \mathrm{ClO} 2$ & 5195.9299 & \\
\hline C165H225N52O103P17 & C11 & $\mathrm{C} 11 \mathrm{H} 10 \mathrm{O} 2$ & 5111.4262 & \\
\hline C163H222N53O103P17 & $\mathrm{C} 12$ & $\mathrm{C} 9 \mathrm{H} 7 \mathrm{NO} 2$ & 5098.3877 & \\
\hline C163H223N52O102P17 & D1 & $\mathrm{C} 9 \mathrm{H} 8 \mathrm{O}$ & 5069.3895 & \\
\hline C161H222N53O104P17 & D2 & C7H7NO3 & 5090.3657 & \\
\hline C164H221FN53O103P17 & D3 & $\mathrm{C} 10 \mathrm{H} 6 \mathrm{~F} 3 \mathrm{NO} 2$ & 5166.3856 & \\
\hline C165H222ClN54O103P17 & D4 & $\mathrm{C} 11 \mathrm{H} 7 \mathrm{ClN} 2 \mathrm{O} 2$ & 5171.8688 & \\
\hline C163H233N52O103P17 & D5 & С9H18O2 & 5085.3889 & \\
\hline
\end{tabular}




\begin{tabular}{|c|c|c|c|c|}
\hline Product_FW & Well & monomer_FW & Prod_MW & DNA_SM \\
\hline C162H225N52O103P17 & D6 & $\mathrm{C} 8 \mathrm{H} 10 \mathrm{O} 2$ & 5075.3941 & \\
\hline C164H225N52O103P17 & D7 & $\mathrm{C} 10 \mathrm{H} 10 \mathrm{O} 2$ & 5099.4155 & \\
\hline C164H232N53O104P17 & D8 & C10H17NO3 & 5136.4772 & \\
\hline C164H223N52O103P17 & D9 & $\mathrm{C} 10 \mathrm{H} 8 \mathrm{O} 2$ & 5097.3996 & \\
\hline C164H222BrN52O103P17 & D10 & $\mathrm{C} 10 \mathrm{H} 7 \mathrm{BrO} 2$ & 5176.2957 & \\
\hline C167H232N54O104P17 & D11 & $\mathrm{C} 13 \mathrm{H} 16 \mathrm{~N} 2 \mathrm{O} 3$ & 5185.508 & \\
\hline C167H225N52O103P17 & D12 & $\mathrm{C} 13 \mathrm{H} 10 \mathrm{O} 2$ & 5135.4476 & \\
\hline C162H220N53O105P17 & E1 & C8H5NO4 & 5116.3599 & \\
\hline C164H224N53O103P17 & E2 & $\mathrm{C} 10 \mathrm{H} 9 \mathrm{NO} 2$ & 5112.4142 & \\
\hline C163H223N52O102SP17 & E3 & C9H8OS & 5101.4545 & \\
\hline $\mathrm{C} 162 \mathrm{H} 222 \mathrm{~N} 53 \mathrm{O} 102 \mathrm{P} 17$ & E4 & $\mathrm{C} 8 \mathrm{H} 7 \mathrm{NO}$ & 5070.3776 & \\
\hline C169H234N53O103P17 & E5 & $\mathrm{C} 15 \mathrm{H} 19 \mathrm{NO} 2$ & 5182.5471 & \\
\hline C167H224ClN54O103P17 & E6 & $\mathrm{C} 13 \mathrm{H} 9 \mathrm{ClN} 2 \mathrm{O} 2$ & 5197.906 & \\
\hline C171H230N53O104P17 & E7 & C17H15NO3 & 5218.5362 & \\
\hline $\mathrm{C} 163 \mathrm{H} 219 \mathrm{Cl} 2 \mathrm{~N} 52 \mathrm{O} 102 \mathrm{P} 17$ & E8 & $\mathrm{C} 9 \mathrm{H} 4 \mathrm{Cl} 2 \mathrm{O}$ & 5136.2637 & \\
\hline C164H226N53O104P17 & E9 & $\mathrm{C} 10 \mathrm{H} 11 \mathrm{NO} 3$ & 5130.4295 & \\
\hline C163H221N52O103P17 & E10 & $\mathrm{C} 9 \mathrm{H} 6 \mathrm{O} 2$ & 5083.373 & \\
\hline C167H230N53O103P17 & E11 & $\mathrm{C} 13 \mathrm{H} 15 \mathrm{NO} 2$ & 5154.494 & \\
\hline C163H221C1N52O103P17 & E12 & $\mathrm{C} 9 \mathrm{H} 6 \mathrm{Cl} 2 \mathrm{O} 2$ & 5154.279 & \\
\hline $\mathrm{C} 122 \mathrm{H} 221 \mathrm{~N} 52 \mathrm{O} 102 \mathrm{P} 17 \mathrm{~S}$ & $\mathrm{~F} 1$ & C8H6OS & 5087.4279 & \\
\hline C165H226N53O103P17 & $\mathrm{F} 2$ & $\mathrm{C} 11 \mathrm{H} 11 \mathrm{NO} 2$ & 5126.4408 & \\
\hline C164H221N52O103P17 & F3 & $\mathrm{C} 10 \mathrm{H} 6 \mathrm{O} 2$ & 5095.3837 & \\
\hline
\end{tabular}




\begin{tabular}{|c|c|c|c|c|}
\hline Product_FW & Well & monomer_FW & Prod_MW & DNA_SM \\
\hline C166H227N52O104P17 & $\mathrm{F} 4$ & $\mathrm{C} 12 \mathrm{H} 12 \mathrm{O} 3$ & 5141.4522 & \\
\hline C164H224N53O104P17 & F5 & $\mathrm{C} 10 \mathrm{H} 9 \mathrm{NO} 3$ & 5128.4136 & \\
\hline $\mathrm{C} 122 \mathrm{H} 222 \mathrm{~N} 53 \mathrm{O} 104 \mathrm{P} 17 \mathrm{~S}$ & F6 & C8H7NO3S & 5134.4414 & \\
\hline C166H224N55O103P17 & F7 & $\mathrm{C} 12 \mathrm{H} 9 \mathrm{~N} 3 \mathrm{O} 2$ & 5164.449 & \\
\hline C164H225N52O104P17S & F8 & C10H10O3S & 5147.4799 & \\
\hline C164H232N53O104P17 & F9 & $\mathrm{C} 10 \mathrm{H} 17 \mathrm{NO} 3$ & 5136.4772 & \\
\hline C167H224CIN54O103P17 & F10 & $\mathrm{C} 13 \mathrm{H} 9 \mathrm{ClN} 2 \mathrm{O} 2$ & 5197.906 & \\
\hline C162H220FN52O102P17 & F11 & C8H5FO & 5073.3534 & \\
\hline C166H229N52O103P17 & F12 & $\mathrm{C} 12 \mathrm{H} 14 \mathrm{O} 2$ & 5127.4686 & \\
\hline C163H222N53O104P17 & G1 & $\mathrm{C} 9 \mathrm{H} 7 \mathrm{NO} 3$ & 5114.3871 & \\
\hline C168H231FN53O104P17 & $\mathrm{G} 2$ & C14H16FNO3 & 5202.5104 & \\
\hline C170H227N52O103P17 & G3 & $\mathrm{C} 16 \mathrm{H} 12 \mathrm{O} 2$ & 5173.4956 & \\
\hline C163H222N53O104P17 & G4 & $\mathrm{C} 9 \mathrm{H} 7 \mathrm{NO} 3$ & 5114.3871 & \\
\hline C163H221N52O104P17 & G5 & $\mathrm{C} 9 \mathrm{H} 6 \mathrm{O} 3$ & 5099.3724 & \\
\hline C163H223N52O103P17 & G6 & $\mathrm{C} 9 \mathrm{H} 8 \mathrm{O} 2$ & 5085.3889 & \\
\hline C165H232N53O104P17 & G7 & $\mathrm{C} 11 \mathrm{H} 17 \mathrm{NO} 3$ & 5148.4879 & \\
\hline C166H228N53O104P17 & G8 & $\mathrm{C} 12 \mathrm{H} 13 \mathrm{NO} 3$ & 5156.4668 & \\
\hline C165H224N53O102P17 & G9 & C11H9NO & 5108.4255 & \\
\hline C168H225N52O103P17 & G10 & $\mathrm{C} 14 \mathrm{H} 10 \mathrm{O} 2$ & 5147.4583 & \\
\hline C165H229N54O104P17S & G11 & $\mathrm{C} 11 \mathrm{H} 14 \mathrm{~N} 2 \mathrm{O} 3 \mathrm{~S}$ & 5191.5357 & \\
\hline C165H227N52O105P17 & G12 & $\mathrm{C} 11 \mathrm{H} 12 \mathrm{O} 4$ & 5145.4409 & \\
\hline C162H228N53O103P17 & H1 & $\mathrm{C} 8 \mathrm{H} 13 \mathrm{NO} 2$ & 5092.4246 & \\
\hline
\end{tabular}




\begin{tabular}{|l|l|l|l|l|}
\hline Product_FW & Well & monomer_FW & Prod_MW & DNA_SM \\
\hline C169H226FN52O102P17 & H2 & C15H11FO & 5163.4759 & \\
\hline C168H225N52O102P17 & H3 & C14H10O & 5131.4589 & \\
\hline C165H225N52O102P17 & H4 & C11H10O & 5095.4268 & \\
\hline C122H226N53O104P17 & H5 & C8H11NO3 & 5106.4081 & \\
\hline C163H223N52O103P17 & H6 & C9H8O2 & 5085.3889 & \\
\hline C164H222N53O102P17S & H7 & C10H7NOS & 5126.464 & \\
\hline C172H228N53O103P17 & H8 & C18H13NO2 & 5212.5316 & \\
\hline C163H222C1N52O102P17 & H9 & C9H7ClO & 5103.8346 & \\
\hline C170H228N53O103P17 & H10 & C16H13NO2 & 5188.5102 & \\
\hline C163H221N54O102P17 & H11 & C9H6N2O & 5095.387 & \\
\hline C164H230N53O103P17 & H12 & C10H15NO2 & 5118.4619 & \\
\hline
\end{tabular}

\section{Abbreviations:}

EDC-HCl, $N$-Ethyl- $N$ '-[3-, (dimethylamino)propyl]carbodiimide hydrochloride; HOPO, 2Hydroxypyridine $\mathrm{N}$-oxide; HATU, 1-[Bis(dimethylamino)methylene]-1 $\mathrm{H}$-1,2,3-triazolo[4,5b] pyridinium 3-oxide hexafluorophosphate; DIEA, $N, N$-Diisopropylethylamine; DBU, 1,8Diazabicyclo[5.4. 0]undec-7-ene; MTBD, 1,3,4,6,7,8-Hexahydro-1-methyl-2H-pyrimido[1,2a]pyrimidine; BTMG, 2-tert-Butyl-1,1,3,3-tetramethylguanidine; BEMP, 2-tert-Butylimino-2diethylamino-1,3-dimethylperhydro-1,3,2-diazaphosphorine; BTTP, tert-Butyliminotri(pyrrolidino)phosphorane; P2-Et, 1-Ethyl-2,2,4,4,4-pentakis(dimethylamino)-2 $\lambda^{5}, 4 \lambda^{5}$ catenadi(phosphazene); dppf Pd G3, Methanesulfonato 1,1-ferrocenediylbis(diphenylphosphino) (2'-amino-1,1'-biphenyl-2-yl) palladium(II); XantPhos Pd G3, [(4,5Bis(diphenylphosphino)-9,9-dimethylxanthene)-2-(2'-amino-1,1'-biphenyl)]palladium(II) methanesulfonate ; Ruphos Pd G3, (2-Dicyclohexylphosphino-2',6'-diisopropoxy-1,1'biphenyl)[2-(2'-amino-1,1'-biphenyl)]palladium(II) methanesulfonate; $t$ BuXPhos-Pd-G3, [(2-Ditert-butylphosphino-2',4',6'-triisopropyl-1,1'-biphenyl)-2-(2'-amino-1,1'-biphenyl)] palladium(II) methanesulfonate; AdBrettPhos Pd G3, [2-(Di-1-adamantylphosphino)-2',4',6'-triisopropyl-3,6dimethoxybiphenyl][2-(2'-amino-1,1'-biphenyl)]palladium(II) methanesulfonate; $t$, BuBrettPhos Pd G3, [(2-Di-tert-butylphosphino-3,6-dimethoxy-2',4',6'-triisopropyl-1,1'-biphenyl)-2-(2'amino-1,1'-biphenyl)]palladium(II) methanesulfonate; BrettPhos Pd G3, [(2-Di- 
cyclohexylphosphino-3,6-dimethoxy-2',4',6'- triisopropyl-1,1'-biphenyl)-2-(2'-amino-1,1' biphenyl)]palladium(II) methanesulfonate methanesulfonate; rac-BINAP-Pd-G3, $( \pm)\left[1,1^{\prime}-\right.$ Binaphthalene]-2,2'-diylbis[diphenylphosphine]palladium(II) methanesulfonate methanesulfonate; $\mathrm{P}(t \text {-Bu })_{3} \mathrm{Pd} \mathrm{G} 3$, [(tri-tert-butylphosphine)]palladium(II) methanesulfonate methanesulfonate; PPA G3, Methanesulfonato(1,3,5,7-tetramethyl-8-phenyl-2,4,6-trioxa-8phosphaadamantane)(2'-amino-1,1'-biphenyl-2-yl)palladium(II); Aphos Pd G3, [4-(Di-tertbutylphosphino)- $N, N$-dimethylaniline-2-(2'-aminobiphenyl)]palladium(II) methanesulfonate; Xphos Pd G3, (2-Dicyclohexylphosphino-2', 4',6'-triisopropyl-1,1'-biphenyl)[2-(2'-amino-1,1'biphenyl)]palladium(II) methanesulfonate; DTBTF Pd G3, [1,1'-Bis(di-tert-

butylphosphino)ferrocene] palladium(II) methanesulfonate methanesulfonate; Josiphos Pd G3, [(R)-1-[(Sp)-2-(Dicyclohexylphosphino)ferrocenyl] ethyldi-tert-butylphosphine][2-(2'-amino1,1'-biphenyl)]palladium(II) methanesulfonate; Mordalphos Pd G3, (2-(Di-1adamantylphosphino)morpholinobenzene)[2-(2'-amino-1,1'-biphenyl)]palladium(II) methanesulfonate; Rockphos Pd G3, [(2-Di-tert-butylphosphino-3-methoxy-6-methyl-2',4',6'triisopropyl-1,1'-biphenyl)-2-(2-aminobiphenyl)]palladium(II) methanesulfonate

\section{Reference:}

1. Bunin, B. A.; Dener, J. M.; Livingston, D. A., Application of combinatorial and parallel synthesis to medicinal chemistry. Annu. Rep. Med. Chem. 1999, 34, 267-286. 